\title{
Joint detection and localization of vessels at sea with a GNSS-based multistatic radar
}

\author{
Fabrizio Santi, Federica Pieralice, Debora Pastina
}

\begin{abstract}
This paper addresses the exploitation of GNSS as opportunistic sources for the joint detection and localization of vessels at sea in a passive multistatic radar system. A single receiver mounted on a proper platform (e.g., a moored buoy) can collect the signals emitted by multiple navigation satellites and reflected from ship targets of interest. This paper puts forward a single-stage approach to jointly detect and localize the ship targets by making use of long integration times (tens of seconds) and properly exploiting the spatial diversity offered by such a configuration. A proper strategy is defined to form a long-time and multistatic range\&Doppler (RD) map, where the total target power can be reinforced with respect to, in turn, the case in which the RD map is obtained over as a short dwell and the case in which a single transmitter is employed. The exploitation of both the long integration time and the multiple transmitters can greatly enhance the performance of the system, allowing counteracting the low power budget provided by the considered sources representing the main bottleneck of this technology. Moreover, the proposed singlestage approach can reach superior detection performance than a conventional two-stage process where peripheral decisions are taken at each bistatic link and subsequently the localization is achieved by multilateration methods. Theoretical and simulated performance analysis is proposed and also validated by means of experimental results considering Galileo transmitters and different types of targets of opportunity in different scenarios. Obtained results prove the effectiveness of the proposed method to provide detection and localization of ship targets of interest.
\end{abstract}

Keywords- multistatic radar, GNSS-based passive radar, ship detection, ship localization, maritime surveillance, long-integration time

\section{INTRODUCTION}

$\mathrm{L}$ AST three decades have seen the alternative utilization of Global Navigation Satellite Systems (GNSS) acquiring an increasing appeal in the remote sensing community. The broadcast signals transmitted by the satellites can be considered as highly-precise synchronized and ubiquitous electromagnetic sources, offering new interesting opportunities for Earth observation and monitoring. Scientists and engineers explored a lot of remote sensing systems and techniques based on the exploitation of the GNSS reflected signal (GNSSReflectometry), bringing to innovative solutions for applications such as ocean altimetry [1], sea state observation [2] and soil moisture estimation [3], just to name a few. In addition, also techniques for the reconstruction of the normalized radar cross section image from GNSS-R Delay and Doppler Map were proposed in [4-5]. As navigation satellites operate in the microwave region (L-band), they have been also considered as illuminators of opportunity for passive radar systems, expanding the range of possible applications. The advent of new generations of GNSS satellites (such as the GPS III), and the finalization of the European Galileo and the Chinese BeiDou constellations, as well as development of satellite-based augmentation systems (such as the Japanese Quasi-Zenith Satellite System), are expected to make the secondary utilization of GNSS a topic of still growing interest in the next years [6].

One of the most well-established GNSS-based radar technologies is the passive Synthetic Aperture Radar (SAR), which has been investigated at both theoretical and practical levels considering different kinds of configurations, comprising ground-based or airborne receivers and bistatic or multistatic geometries [7]-[15]. Moreover, detection of moving targets has been addressed, particularly for air targets such as airplanes and helicopters by considering forward-scattering radar modes [16]-[18].

A quite new research direction for this technology is its application to maritime surveillance, where it appears to be a prospective and very attractive solution. Indeed, it benefits of the peculiarities of passive radar systems, such as costeffectiveness, absence of harmful radiations, covertness and anti-stealth capabilities [19]. Moreover, GNSS satellites offer a global coverage, thus potentially enabling permanent monitoring of both coastal and open sea areas, being the latter out of the coverage of systems relying on terrestrial illuminators such as active Vessel Traffic System (VTS) and passive DVB$\mathrm{T}$-based radars. The main drawback stays in the fact that navigation satellites are very low EIRP sources, providing a flux power density on the sea level around $3 \times 10^{-14} \mathrm{~W} / \mathrm{m}^{2}[8]$, making challenging the detection of the ship targets of interest.

In recent researches, the feasibility of this novel application of GNSS signals has been investigated in [20]-[22] using GNSS-Reflectometry. Moving to the radar ambit, in [23] the feasibility of maritime Moving Target Indication (MTI) by using a GNSS-based passive radar has been investigated and experimentally verified considering large vessels [namely, high radar cross section (RCS) targets] at relatively short receiver standoffs: bright spots were observed in the bistatic range\&Doppler (RD) maps obtained over short time intervals (2-3 seconds). To counteract the restricted power budget provided by GNSS and enhancing the detection performance of the system, advanced MTI modes have been defined in [24]. These considered the integration of the reflected signals for long dwells (tens of seconds) by properly correcting the target range and Doppler migration. The experimental results therein 
provided showed the effectiveness of the long-integration time techniques at enabling MTI for ships undetectable with the conventional (i.e., short integration time) algorithms and, at the same time, increasing the detection range.

Despite the increased performance enabled by the long integration times, detection of low RCS and/or far targets still represents a main technical challenge. In addition, in the abovementioned works a bistatic system configuration comprising a single satellite and a single receiver has been considered. Such a configuration suffers for a poor localization accuracy, limited to the locus of points given by the intersection of the bistatic range ellipses with the area illuminated by the receiver antenna. In order to increase the angular resolution of a bistatic system, two solutions could be considered. The former is using a rotating antenna beam. However, the achievable dwells with such a configuration contrast with the requirement of long integration time, so that such a solution appears to be not feasible in the considered scenario. The second solution is exploiting an array receiver configuration (see for example [25], addressing a Fractional Fourier Transform-based technique for an array receiver exploiting low-power satellite illuminators), however this entails higher costs for the receiver development, partially compromising the cost-effectiveness of the proposed technology.

Apart the global and persistent coverage enabled by GNSS, the unique feature of these systems is that they operate with large constellations. A single completely operative constellation guarantees that 4-8 satellites are simultaneously in view of each point over the Earth 'surface; therefore, up to 32 satellites may illuminate at the same time any sea zone by considering the global systems (GPS, GLONASS, Galileo and BeiDou) in full capacity; this number could be also increased by considering the forthcoming satellite-based augmentation systems. Remarkably, GNSS operate with code (or frequency) division multiple access schemes, so that a single receiver inherently forms a passive multistatic radar system. The multistatic configuration offers a number of benefits with respect to its bistatic (i.e., single satellite) counterpart. For example, in GNSS-based SAR imagery it has been shown that multi-perspective acquisitions can drastically improve the spatial resolution [11],[14] and enhance image information [13]. With respect to the maritime surveillance application, the availability of multiple emitters brings to three main added values: i) the possibility to estimate the ship Direction-ofArrival (DOA) without having to consider multi-beam or array receiving systems; ii) the possibility to collect data samples over multiple bistatic links, potentially improving the system power budget; iii) the exploitation of a large spatial diversity, greatly alleviating the deleterious effects of target RCS scintillations [26].

Recently, a multistatic system has been considered in [27], where it has been demonstrated that vessels at sea can be simultaneously detected at multiple bistatic links exploiting multiple satellites (even belonging to different constellations) and that their instantaneous locations can be extracted by means of multilateration approaches ([28]). In particular, up to twelve GNSS emitters were exploited and it was shown that sufficient localization accuracy for practical purposes can be achieved. Nevertheless, it should be pointed out that even though this approach has been proved to be effective in target localization, it requires the targets to be detected at the individual bistatic links level, namely the available spatial diversity is not directly exploited for detection purposes.

In this work, we consider a multistatic system and we propose a novel approach for the exploitation of such configuration in order to provide a joint detection and localization of the ship targets. Particularly, we consider a centralized detection method that first integrates the signals concerning the different bistatic links to increase the signal-tonoise ratio (SNR) level and then implements a single-stage to detect and possibly localize the target. Thanks to the achievement of the spatial diversity gain [29], the single-stage approach may potentially outperform the conventional twostage process where independent decisions are taken at each bistatic link and subsequently the localization is performed. To achieve such a purpose, the signals collected by a single receiver over long dwell times and over multiple baselines are integrated in both the temporal and space domains to form a long-time and multistatic RD map. Such a map is obtained through a non-coherent combination of the RD maps achieved over short and consecutive Coherent Processing Intervals (CPIs) over all the available baselines. If the target energy is properly integrated over the individual RD maps, the total target power can be effectively increased, while at the same time the disturbance background fluctuations reduced thus improving the final SNR.

To properly combine the contributions over the long dwells and the multiple baselines, ad hoc range\&Doppler migration compensation techniques as well as time\&space integration strategies need to be applied. Concerning migration compensation, two sources have to be taken into account. The former is a migration over the temporal domain, i.e. the range and Doppler migration that the target experiences in each baseline due to its motion. The latter is a migration over the space domain, due to the different RD positions observed in the multiple bistatic geometries. To cope with these problems, a new (time\&space) migration compensation technique is proposed that realizes an energy alignment among the RD maps pertaining the successive CPIs at each individual baseline and remaps the different bistatic range and Doppler planes into a common monostatic equivalent plane. It should be noted that the time migration compensation technique considered in [24] can be viewed as a particular case of the (time\&space) technique here proposed. A preliminary version of the proposed compensation technique was presented in [30] and tested against few experimental datasets in [31]. Regarding the integration over the time and space domains, firstly an optimum technique is derived and then a suboptimum approach is also considered. For both approaches the theoretical performance, in terms of false alarm and detection probability, are derived, investigated and compared analyzing the increase available from the exploitation of the time and/or space domains and showing that the suboptimum scheme yields limited losses in many cases of practical interest. In addition, since the mapping of the target bistatic range and Doppler history in the equivalent monostatic plane depends on the target DOA, a bank structure is adopted being each branch of the bank matched to a specific DOA value. The information provided by the bank is thus exploited to estimate the target DOA and range and DOA information are then used to localize the detected target. 


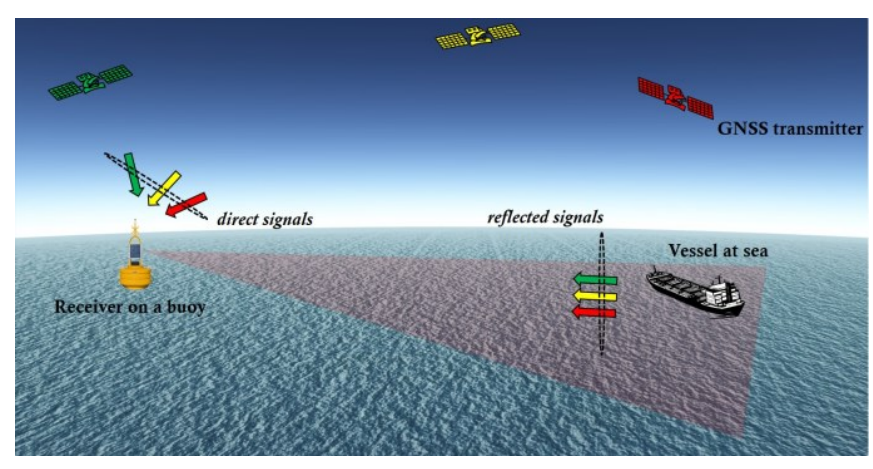

Fig. 1 System concept of GNSS-based multistatic radar for maritime surveillance.

Criteria to design the bank are provided and localization capabilities are investigated and proved by means of synthetic datasets. Finally, results from experimental campaigns (using up to four Galileo transmitters) are reported and discussed clearly demonstrating the effectiveness of the proposed approach.

The remainder of the paper is organized as follows. Section II describes the operative conditions of the system and the received signal model, while the multistatic detection and localization technique is presented in Section III. Performance analysis is provided in Section IV and experimental results are presented in Section V. Section VI closes the paper. Analytical details are reported in the Appendices.

\section{Operative Conditions AND Signal Model}

The operative conditions of the systems are given by navigation satellites as transmitters of opportunity and a receive-only device in a remote location above the sea (e.g., it could be mounted on a moored buoy or on the coast, see Fig. 1). As usually in GNSS-based passive radar systems, the receiver operates with two RF channels [7]. The former is the reference channel, equipped with a low-gain antenna pointed toward the sky and recording the direct signals for the synchronization between transmitters and receiver. The latter is the surveillance channel, operating with a high-gain antenna pointed toward the surveyed area and collecting the signal reflections. The targets of interest are ships moving in the footprint of the surveillance antenna that the system aims at detecting and localizing.

The remainder of the section defines the system geometry pertaining to this scenario assuming a stationary receiver (subsection A) and provides the derived received signal model (subsection $\mathrm{B}$ ).

\section{A. Acquisition geometry}

Fig. 2 shows the local $(O, X, Y, Z)$ reference system, which is, without loss of generality, centered in the receiver position and with the $X$-axis defining its line-of-sight (LOS), i.e. the pointing direction of the surveillance antenna. Let $M$ be the number of satellites in visibility and let $T_{a}$ be the aperture time. Since the focus is on ship targets and a flat Earth geometry can be assumed (because of the relatively small size of the observed area), the height of the target can be neglected. Let $\boldsymbol{T} \boldsymbol{X}_{m}=$ $\left[x_{m}, y_{m}, z_{m}\right]^{T}, \boldsymbol{R} \boldsymbol{X}=[0,0,0]^{T}$ and $\boldsymbol{T} \boldsymbol{G}=\left[x_{t}, y_{t}, 0\right]^{T}$ denote the $m$ th transmitter, receiver and target instantaneous positions, respectively. The paths $m$ th transmitter to target, target to

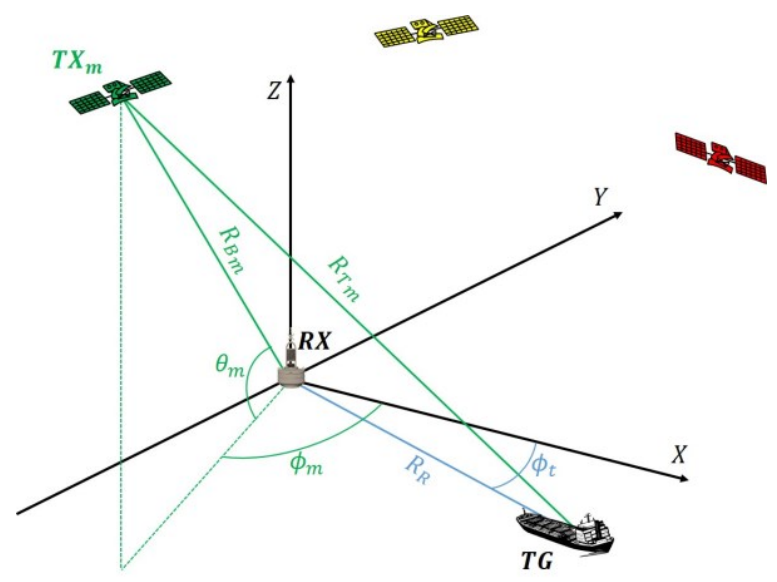

Fig. 2 System geometry.

receiver, and $m$ th transmitter to receiver (i.e., the $m$ th baseline) are therefore $R_{T_{m}}=\left|\boldsymbol{T} \boldsymbol{X}_{m}-\boldsymbol{T} \boldsymbol{G}\right|, \quad R_{R}=|\boldsymbol{T} \boldsymbol{G}-\boldsymbol{R} \boldsymbol{X}|$, and $R_{B_{m}}=\left|\boldsymbol{T} \boldsymbol{X}_{m}-\boldsymbol{R} \boldsymbol{X}\right|$, respectively. Further, it is useful to define the following angles. $\phi_{m}$ is the clockwise angle on the ground plane $(Z=0)$ between the radar LOS and the $m$ th satellite projection on the ground plane; $\theta_{m}$ is the out-of-plane angle between the ground plane and the $m$ th satellite; finally, $\phi_{t}$ is the clockwise angle on the ground plane between the radar LOS and the target, namely the target DOA. Therefore, the transmitter and target coordinates can be written as

$$
\begin{gathered}
\boldsymbol{T}_{m}=\left[\begin{array}{c}
R_{B_{m}} \cos \theta_{m} \cos \phi_{m} \\
R_{B_{m}} \cos \theta_{m} \sin \phi_{m} \\
R_{B_{m}} \sin \theta_{m}
\end{array}\right] \\
\boldsymbol{T} \boldsymbol{G}=\left[\begin{array}{c}
R_{R} \cos \phi_{t} \\
R_{R} \sin \phi_{t} \\
0
\end{array}\right]
\end{gathered}
$$

\section{B. Received signal model}

Despite GNSS signals are continuous in time, the received data can be formatted in a 2D scheme by defining an equivalent Pulse Repetition Interval $T_{c}$ that can be matched to the ranging code length (typically, $1 \mathrm{~ms}$ ). Therefore, fast-time and slow time variables can be defined as $\tau \in\left[0, T_{c}\right]$ and $u \in\left[-\frac{T_{a}}{2}, \frac{T_{a}}{2}\right]$, respectively.

A signal synchronization algorithm tracks the direct signals parameters, i.e. time delay, Doppler and phase [23]; the navigation message (if one exists) is also tracked by detecting the $\pm \pi$ phase transition due to the navigation code bit inversion [7]. The synchronization enables the generation of noise-free replicas of the $M$ references signals, which are exploited to perform the range compression. Taking also into account the orthogonality of the PRN codes pertaining the different satellites, with cross-correlation terms that are expected being well below the noise floor of the receiver, the fast-time and slow-time $(\tau, u)$ radar data pertaining the moving target at the mth baseline $(m=1, \ldots, M)$ can be expressed as

$$
\begin{aligned}
s_{m}(\tau, u)= & A_{m}(u) \cdot \exp \left\{-j \frac{2 \pi}{\lambda} R_{m}(u)\right\} . \\
& \Lambda\left[\tau-\frac{R_{m}(u)}{c}\right] \cdot \operatorname{rect}\left[\frac{u}{T_{a}}\right]
\end{aligned}
$$


where $\operatorname{rect}(\mathrm{x})=1$ for $|x|<1 / 2$ and 0 otherwise. $R_{m}(u)$ is the instantaneous bistatic range, given by the range difference between the reflected and direct signals:

$$
R_{m}(u)=R_{T_{m}}(u)+R_{R}(u)-R_{B_{m}}(u)
$$

$c$ is the speed of light, $\lambda$ is the wavelength and $\Lambda[\cdot]$ is the crosscorrelation function between the reference and surveillance signal. It is worth to point out that GNSS operates with different signals and with different frequency bands. Therefore, in the more general case $\lambda$ and $\Lambda$ should vary with $m$. However, for any GNSS satellite, $\Lambda[\cdot]$ can be well approximated by a triangular function with a pulsewidth twice that of the code chip duration (whose inverse defines the signal bandwidth, hereinafter denoted as B) [11], [12]. For sake of simplicity, in this work we assume the $M$ signals sharing the same carrier frequency and the same value of B, e.g. Galileo E5a and GPS L5 signals.

Finally, $A_{m}(u)$ represents the complex amplitude backscattered by the target when illuminated by the $m$ th satellite. Since the coherence of the target response is not expected to be maintained over the long aperture times considered, $A_{m}$ changes with time. In the considered scenario, suitable values for which ship target RCS can be considered constant are in the order of 2-3 sec [23][24][27]. As it will be detailed later on, the proposed technique realizes a noncoherent integration of consecutive coherent processing intervals (CPIs), hence it is useful to consider the whole aperture time segmented in consecutive time intervals (hereinafter named frames) of duration $T_{f}$ within which constant target RCS should normally be achieved. Let $N$ be the number of considered frames and, without loss of generality, let us assume $N=\frac{T_{a}}{T_{f}}$. By converting the fast-time dimension into bistatic range as $r=c \cdot \tau$, the range\&slow-time $(r, u)$ data pertaining the $n$th $\left(n=-\frac{N}{2}, \ldots, \frac{N}{2}-1\right)$ frame and the $m$ th satellite can be written as

$$
\begin{array}{r}
s_{m, n}(r, u)=A_{m, n} \cdot \exp \left\{-j \frac{2 \pi}{\lambda} R_{m}(u)\right\} . \\
\Lambda\left[r-R_{m}(u)\right] \cdot \operatorname{rect}\left[\frac{u-n T_{f}}{T_{f}}\right]
\end{array}
$$

Further, by defining the temporal variable $u^{\prime}=u-n T_{f}$ that spans the single frame, (4) can be rewritten as

$$
\begin{gathered}
s_{m, n}\left(r, u^{\prime}\right)=A_{m, n} \cdot \exp \left\{-j \frac{2 \pi}{\lambda} R_{m}\left(u^{\prime}+n T_{f}\right)\right\} . \\
\Lambda\left[r-R_{m}\left(u^{\prime}+n T_{f}\right)\right] \cdot \operatorname{rect}\left[\frac{u^{\prime}}{T_{f}}\right]
\end{gathered}
$$

The bistatic range (3) can be expanded in Taylor series around the reference time instant pertaining the reference frame. Assuming not maneuvering ships, a second order expansion generally suffices, leading to

$$
R_{m}(u) \approx R_{m}^{0}+\dot{R}_{m} u+\ddot{R}_{m} \frac{u^{2}}{2}
$$

Therefore

$$
\begin{gathered}
R_{m}\left(u^{\prime}+n T_{f}\right)=R_{m}^{0}+\dot{R}_{m} u^{\prime}+\dot{R}_{m} n T_{f}+\ddot{R}_{m} \frac{u^{\prime^{2}}}{2}+ \\
\ddot{R}_{m} n T_{f} u^{\prime}+\ddot{R}_{m} \frac{\left(n T_{f}\right)^{2}}{2}
\end{gathered}
$$

By setting

$$
R_{m, n}=R_{m}^{0}+\dot{R}_{m} n T_{f}+\ddot{R}_{m} \frac{\left(n T_{f}\right)^{2}}{2}
$$

(5) can be rewritten as

$$
\begin{aligned}
& s_{m, n}\left(r, u^{\prime}\right)= A_{m, n} \cdot \\
& \exp \left\{-j \frac{2 \pi}{\lambda}\left[R_{m, n}+\left(\dot{R}_{m}+\ddot{R}_{m} n T_{f}\right) u^{\prime}+\ddot{R}_{m} \frac{u^{\prime 2}}{2}\right]\right\} . \\
& \Lambda\left[r-R_{m, n}\right] \cdot \operatorname{rect}\left[\frac{u^{\prime}}{T_{f}}\right]
\end{aligned}
$$

where the range walk $\left(\dot{R}_{m} u^{\prime}\right)$ and the range curvature $\left(\ddot{R}_{m} \frac{u^{\prime 2}}{2}\right)$ within the individual frame have been neglected taking into account the typical velocities of the targets of interest (not higher than 20-30 kn) and the values of the range resolution (not better than $15 \mathrm{~m}$ for the Galileo E5a/b or GPS L5 signals).

It is also worth to point out that from (6), the target bistatic Doppler history is obtained as

$$
f_{u_{m}}=-\frac{1}{\lambda}\left[\dot{R}_{m}+\ddot{R}_{m} u\right]=f_{d c_{m}}+f_{d r} u
$$

where $f_{d c_{m}}$ denotes the bistatic Doppler position pertaining the $m$ th baseline at the reference instant, and $f_{d r}$ denotes the target Doppler rate; in the latter, the dependence on index $m$ has been neglected taking into account the large distance between the transmitters and both the receiver and target (namely, $\ddot{R}_{m} \approx$ $\ddot{R} \forall m$ ), [32]. Overall, the range-compressed\&slow-time data pertaining the $n$th frame and the $m$ th satellite are given by

$$
\begin{gathered}
s_{m, n}\left(r, u^{\prime}\right)=A_{m, n} \cdot \\
\exp \left\{-j \frac{2 \pi}{\lambda}\left[R_{m, n}-\lambda\left(f_{d c_{m}}+f_{d r} n T_{f}\right) u^{\prime}-\lambda f_{d r} \frac{u^{\prime 2}}{2}\right]\right\} \\
\cdot \Lambda\left[r-R_{m, n}\right] \cdot \operatorname{rect}\left[\frac{u^{\prime}}{T_{f}}\right]
\end{gathered}
$$

Based on the signal model above, the proposed processing chain detailed in the following section aims at maximize the SNR available, in order to i) strengthen the target energy sufficiently to enable its detection and ii) jointly provide its localization.

\section{JOINT TARGET DETECTION AND LOCALIZATION WITH MULTISTATIC RADAR}

This section introduces the proposed processing chain that aims at performing an integration over the full aperture $T_{a}$ and over all the $M$ baselines. The block diagram of the proposed technique is depicted in Fig. 3. The final goal is to obtain an integrated RD map, where the target can be detected and jointly localized in the Cartesian plane. The main idea is to obtain a $\mathrm{RD}$ map for each frame and for each baseline and then to perform the integration. To achieve these purposes, the range\&slow-time data (11) undergo a processing comprising three main stages: target motion compensation, multistatic compensation, and space-time integration, as described in the following.

We explicitly point out that the first stage has been investigated in [24] considering a single baseline, thus the 
proposed technique can be regarded in first instance as a proper generalization of the long time maritime MTI technique. However, as aforementioned, the exploitation of multiple bistatic geometries may not only increase the SNR, but also enables the target localization. Particularly, both the long-time and multiple baselines integrations will allow to improve the SNR and the spatial diversity will enable the target localization.

\section{A. Target Motion Compensation}

Let us focus on the stream of data pertaining the $m$ th baseline. To move from the range/slow-time domain to the range\&Doppler domain, a slow-time Fast Fourier Transform (FFT) could be applied to the individual frame and the achieved $N$ RD maps could be subsequently integrated to form a longtime bistatic RD map. Nevertheless, the target motion implies a misalignment among the achievable maps. Indeed, the target position at the $n$th frame time is $\left(R_{m, n}, f_{d c_{m}}+f_{d r} n T_{f}\right)$, namely, it depends on the frame index $n$. Moreover, from the model in (11) it can be observed that the Doppler frequency changes linearly within the individual frame according to a slope given by the Doppler rate $f_{d r}$, so that losses of the coherent integration gain can arise due to the fact that the signal moves through the Doppler filters. The target motion compensation (TMC) aims at compensating the range\&Doppler migration experienced by the target over the different frame intervals, therefore aligning the energy in the RD position occupied by the target at the reference time instant ( $u=0$ in our case) and at the same time correcting the Doppler spread inside the individual frame, thus maximizing the coherent integration gain. The implementation of the TMC consists in the following steps.

a. Doppler migration compensation - Doppler migration occurs both among the frames and at the single frame level. From (11), the events are respectively described by the laws

$$
\begin{gathered}
\Delta f_{n}=f_{d r} n T_{f} \\
\delta f=f_{d r} u^{\prime}
\end{gathered}
$$

Both these migrations can be corrected by multiplying the range/slow-time data for a phase term accounting both the sources of migration. Then, by means of a slow time FFT, the $n$th RD map is obtained where the target energy has been completely accumulated in the Doppler domain and locates in $\left(R_{m, n}, f_{d c_{m}}\right)$.

b. Range migration compensation - From (8), the range migration among the different frames is described by

$$
\begin{aligned}
\Delta R_{n}= & \dot{R}_{m} n T_{f}+\ddot{R}_{m} \frac{\left(n T_{f}\right)^{2}}{2}= \\
& -\lambda\left(f_{d c_{m}} n T_{f}+f_{d r} \frac{\left(n T_{f}\right)^{2}}{2}\right)
\end{aligned}
$$

Therefore, it is composed by a range walk among the frames, depending on the target Doppler centroid, and by a range curvature depending on the Doppler rate. To correct such migrations: 1) a FFT in the range dimension is applied, thus transiting in the fast frequency/Doppler $\left(f_{r}, f_{u}\right)$ domain; 2) a linear phase term dependent on the Doppler value of the corresponding Doppler cell and a quadratic

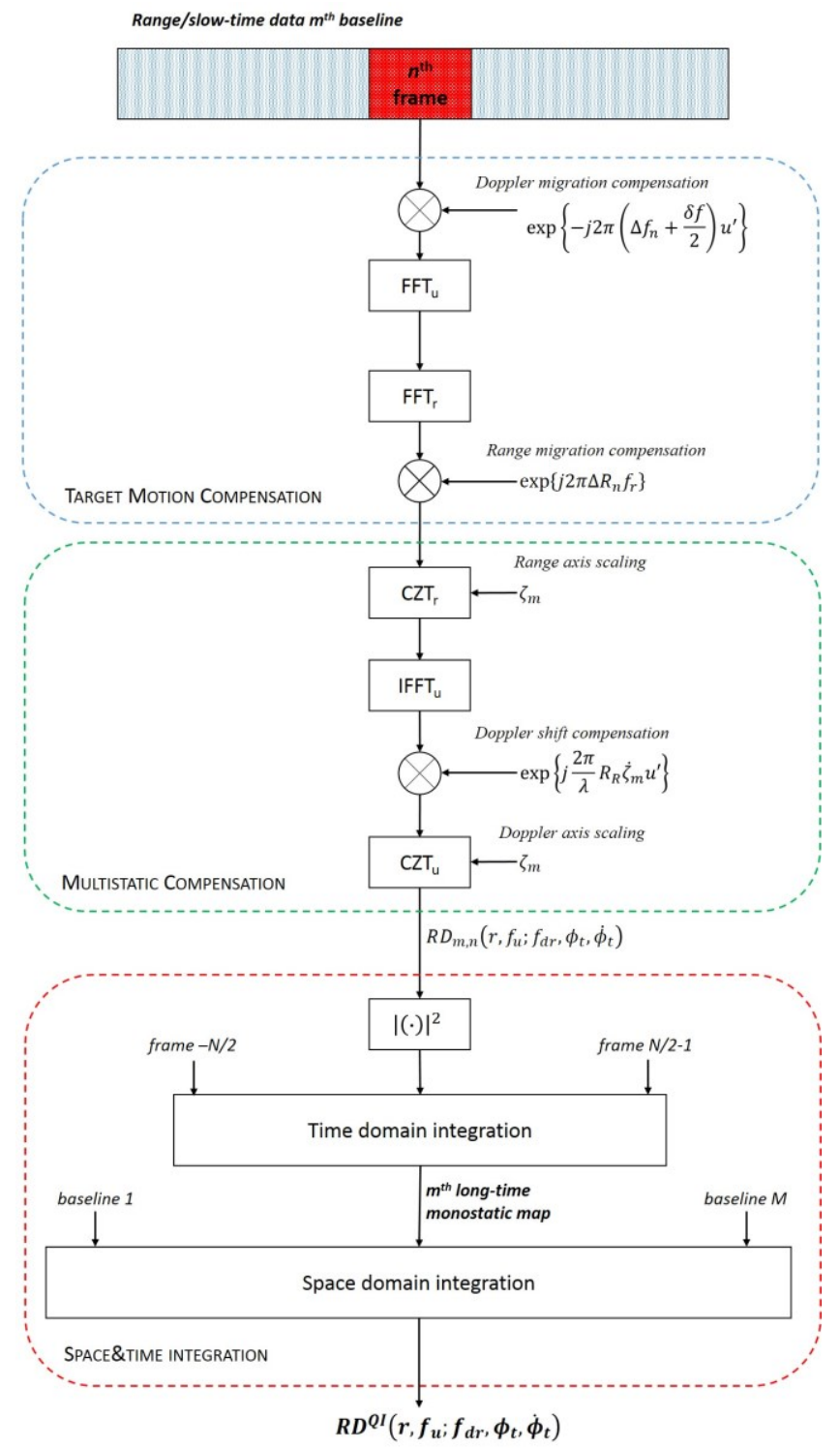

Fig. 3 Processing chain.

phase term depending on the Doppler rate are applied, relocating the target energy in the reference range position $R_{m}^{0}$.

It is interesting to note that the TMC is identically obtained for all the $\mathrm{M}$ bistatic links. Indeed, Doppler migration depends only on the Doppler rate, while the range migration depends on both target Doppler and Doppler rate. Since the Dopplerdependent term of the range migration can be adaptively compensated by varying the Doppler centroid in the $\left(f_{r}, f_{u}\right)$ domain, the whole procedure depends only on the target Doppler rate that is the same for all the baselines, (10). Since its value is unknown, a completely adaptive TMC can be obtained by relying on a bank structure with a proper set of Doppler rate values [24]. In particular, an interval $\left[-f_{d r_{M}},+f_{d r_{M}}\right]$ can be set according to the maximum Doppler rate $f_{d r_{M}}$ one may expect. This corresponds to a target moving with the maximum considered tangential speed at the minimum range. Let $\delta f_{d r}$ be the step size of this interval, to guarantee an uncompensated Doppler migration lower than the Doppler resolution cell (i.e., $1 / \mathrm{T}_{\mathrm{f}}$ ), the following constrain must be fulfilled: 


$$
\delta f_{d r} \leq \frac{1}{N T_{f}^{2}}
$$

At this point (i.e. at the output of the range migration compensation step in Fig. 3), assuming the actual value of the Doppler rate employed in the procedure, the target energy is correctly aligned over the frames domain. If an inverse FFT over the fast frequency domain would be applied, RD maps could be obtained as

$$
\begin{aligned}
S_{m, n}\left(r, f_{u}\right)=A_{m, n} \cdot \Lambda & {\left[r-R_{m}^{0}\right] \cdot } \\
& \operatorname{sinc}\left[\pi\left(f_{u}-f_{d c_{m}}\right) \frac{1}{T_{f}}\right]
\end{aligned}
$$

As it is apparent, target locates in $\left(R_{m}^{0}, f_{d c_{m}}\right)$ in these maps, namely target location is independent from frame index but still dependent on the specific bistatic RD plane, therefore preventing the possibility to integrate over space domain. The alignment of the target energy over the multiple bistatic links is referred to as Multistatic Compensation (MSC) detailed in the following sub-section. As depicted in Fig. 3, this stage is fed with the motion compensated maps in the fast frequency/Doppler domain.

\section{B. Multistatic Compensation}

The goal of the MSC is to map the target energy in a domain that does not depend on the specific bistatic geometry, namely on the index $m$. Such a domain is the target-to-receive range\&Doppler plane, namely the domain pertaining the targetto-receive distance $R_{R}$ (hereinafter referred to as monostatic range) and the related Doppler frequency $f_{u_{R}}=-\frac{1}{\lambda} \dot{R}_{R}$ (hereinafter, monostatic Doppler).

With this objective in mind, we first rewrite the bistatic range (3) as $R_{m}(u)=R_{R}(u)+\Delta R_{m}(u)$, where the term $\Delta R_{m}(u)=R_{T_{m}}(u)-R_{B_{m}}(u)$ represents the transmitter contribution to the bistatic range history of the target. Then, $\Delta R_{m}(u)$ is expanded in Taylor series around the receiver position, arresting the series at the first order, carrying to (the dependence on the slow-time has been hereinafter omitted for sake of simplicity)

$$
\begin{aligned}
\Delta R_{m} \approx- & \frac{x \cdot x_{m}+y \cdot y_{m}}{\sqrt{x_{m}^{2}+y_{m}^{2}+z_{m}^{2}}}= \\
& -R_{R} \cdot\left[\cos \left(\theta_{m}\right) \cos \left(\phi_{m}-\phi_{t}\right)\right]
\end{aligned}
$$

Therefore, $R_{m}$ can be approximated as

$$
\begin{gathered}
R_{m} \approx R_{R} \cdot\left[1-\cos \left(\theta_{m}\right) \cos \left(\phi_{m}-\phi_{t}\right)\right] \\
=R_{R} \cdot \zeta_{m}\left(\phi_{t}\right)
\end{gathered}
$$

where $\zeta_{m}\left(\phi_{t}\right)$ is a target DOA-dependent scale factor. Hence, the bistatic Doppler is easily obtained as

$$
f_{u_{m}} \approx f_{u_{R}} \cdot \zeta_{m}\left(\phi_{t}\right)-\frac{R_{R}}{\lambda} \cdot \dot{\zeta}_{m}\left(\phi_{t}, \dot{\phi}_{t}\right)
$$

where $\dot{\phi}_{t}$ is the rate of the target DOA and

$$
\begin{gathered}
\dot{\zeta}_{m}\left(\phi_{t}, \dot{\phi}_{t}\right)=\left[\sin \left(\theta_{m}\right) \cos \left(\phi_{m}-\phi_{t}\right)\right] \cdot\left(\dot{\theta}_{m}+\dot{\phi}_{m}\right) \\
-\left[\cos \left(\theta_{m}\right) \sin \left(\phi_{m}-\phi_{t}\right)\right] \cdot \dot{\phi}_{t}
\end{gathered}
$$

Based on (18) and (19), the MSC converts the bistatic range in the monostatic range and the bistatic Doppler in the monostatic Doppler. To achieve this purpose, three steps are implemented as follows.

a. Range axis scaling - Eq. (18) shows that the bistatic range is a scaled version of the monostatic range with a scale factor depending on the target DOA. Thus, the bistatic range can be converted into the monostatic one by means of a stretching (or shrinkage) of the bistatic range axis by a factor equal to $\zeta_{m}\left(\phi_{t}\right)$. This can be efficiently implemented by making use of the Chirp Zeta Transform (CZT) [33].

b. Doppler shift compensation - From (19) it can be observed that the bistatic Doppler is approximately a DOA-dependent scaled version of the monostatic Doppler plus a shift depending on the target distance from the receiver, its DOA and its DOA rate. The Doppler shift can be compensated by operating a slow-time IFFT to transit in the monostatic range and slow-time domain and multiplying in this domain the data pertaining each range cell for a phase term according to $-\frac{R_{R}}{\lambda} \cdot \dot{\zeta}_{m}\left(\phi_{t}, \dot{\phi}_{t}\right)$.

c. Doppler axis scaling - After the Doppler shift compensation, the bistatic Doppler axis can be converted to monostatic Doppler by resorting again to the CZT with $\zeta_{m}\left(\phi_{t}\right)$ as scale factor.

It has to be noted that while the frame alignment depends on the Doppler rate $f_{d r}$, the MSC depends on the target DOA $\phi_{t}$ and its rate $\dot{\phi}_{t}$. Likewise the TMC, a completely adaptive MSC procedure needs to resort to a filter bank structure with different values of these parameters. The minimum and maximum DOA are defined according to the surveillance antenna beam, while the sampling of the DOA axis has to be chosen in order to ensure a residual misalignment in range and Doppler less than the range and Doppler resolution cell, respectively. The requirement in range results in the following constraint:

$$
\delta \phi_{t} \leq \frac{c}{B R_{R}} \frac{\left(1-\cos \theta_{m} \cos \left(\phi_{m}-\phi_{t}\right)\right)}{\cos \theta_{m} \sin \left(\phi_{m}-\phi_{t}\right)}
$$

while the condition in Doppler gives the following sampling step:

$$
\delta \phi_{t} \leq \frac{1}{T_{f} f_{d c}} \frac{\left(1-\cos \theta_{m} \cos \left(\phi_{m}-\phi_{t}\right)\right)}{\cos \theta_{m} \sin \left(\phi_{m}-\phi_{t}\right)}
$$

The more stringent condition provides the appropriate sampling step.

The bounds on the interval $\left[-\dot{\phi}_{t_{\text {max }}}, \dot{\phi}_{t_{\max }}\right]$ can be set according to the expected maximum rate of change of the target DOA, which can be set according to the maximum tangential velocity and the minimum target-to-receiver distance. The sampling step of $\dot{\phi}_{t}$ has to assure a residual Doppler misalignment lower than the resolution cell $\left(1 / T_{f}\right)$. Starting from this assumption, the constraint is given by:

$$
\delta \dot{\phi}_{t} \leq \frac{\lambda}{T_{f} \cos \theta_{m} \sin \left(\phi_{m}-\phi_{t}\right) R_{R}}
$$

For the $n$th frame and $m$ th link, the RD map following the TMC and MSC procedures for a specific tried $\left(f_{d r}, \phi_{t}, \dot{\phi}_{t}\right)$ is denoted as $R D_{m, n}\left(r, f_{u} ; f_{d r}, \phi_{t}, \dot{\phi}_{t}\right)$. As it will be explained in the next section, the combination of the MN maps allows achieving a space\&time integration gain that is a function of 
these parameters, thus enabling the estimation of detected target location.

\section{Space\&Time Integration}

After the TMC and the MSC, the $R D_{m, n}$ maps resulting from a given branch of the bank have to be integrated into an individual map where the detection procedure can take place. Thus, the question arises as how to combine the maps pertaining to the different frames and baselines. To answer the question, we can derive a combination criteria based on a Likelihood Ratio Test (LRT) by formulating a usual detection problem as the choice between null hypothesis $\mathcal{H}_{0}$ (target does not exist at the selected range and Doppler cell) and alternative hypothesis $\mathcal{H}_{1}$ (target does exist at the selected range and Doppler cell). Let $r_{m, n}$ be the hypothesized range and Doppler cell pertaining the $n$th frame and the $m$ th bistatic channel after that the data have been time- and space- aligned (namely, we are focusing on the $R D_{m, n}$ maps pertaining the actual $\left.f_{d r}, \phi_{t}, \dot{\phi}_{t}\right)$. The binary hypothesis test can be written as

$$
\begin{aligned}
& \mathcal{H}_{0}: r_{m, n}=w_{m, n} \\
& \mathcal{H}_{1}: \quad r_{m, n}=A_{m, n}+w_{m, n}
\end{aligned}
$$

$w_{m, n}$ is the disturbance background. In this work, it is modeled as white Gaussian characterized by power $\sigma_{w}^{2}$, namely $w_{m, n} \sim \mathcal{C} \mathcal{N}\left(0, \sigma_{w}^{2}\right)$ where $\mathcal{C N}$ denotes the complex normal distribution. This assumption can be justified taking into account the very low power budget provided by GNSS satellites, making the system mainly noise-limited rather than clutter limited [24]. It could be also shown that this hypothesis is in agreement with the experimental data presented in Section $\mathrm{V}$.

$A_{m, n}$ represents the complex amplitude of the signal (for the sake of simplicity and without loss of generality, the deterministic improvement factor pertaining the coherent integration gain has been here included in $A_{m, n}$ ). At the individual baseline level, its variation among the different frames is assumed to follow a Swerling I model. Let $\sigma_{m}^{2}$ be target mean power observed at the $m$ th bistatic link, $A_{m, n}$ can be modeled as a zero-mean complex normal random variable with variance $\sigma_{m}^{2}$, i.e., $A_{m, n} \sim \mathcal{C} \mathcal{N}\left(0, \sigma_{m}^{2}\right)$. As a consequence of the different perspectives provided by different satellites, $\sigma_{m}^{2}$ generally varies among the different baselines (i.e., it varies with $m$ ).

From the above model, the likelihood ratio test (LRT) detector $\mathcal{L}$ can be derived as shown in Appendix A, leading to

$$
\mathcal{L}=\sum_{m}\left\{\eta_{m} \sum_{n}\left|r_{m, n}\right|^{2}\right\} \underset{\mathcal{H}_{0}}{\stackrel{\mathcal{H}_{1}}{\gtrless}} t_{h}^{L R T}
$$

where $t_{h}^{L R T}$ is the threshold set by the desired probability of false alarm,

$$
\eta_{m}=\frac{S N R_{m}}{S N R_{m}+1}
$$

and $S N R_{m}$ is the ratio $\sigma_{m}^{2} / \sigma_{w}^{2}$. Eq. (25) shows that $\mathcal{L}$ is the cascade of a quadratic integrator over the frame domain $\left(\sum_{n}\left|r_{m, n}\right|^{2}\right.$ ) followed by a weighted sum over the multiple baselines according to a coefficient $\eta_{m}$ depending on the local
SNR. As it could be expected, greater weight has to be applied to the best baseline in terms of SNR, so, ultimately, to the bistatic geometry observing the higher bistatic target RCS.

It can be observed that although a quadratic integrator is applied at both the temporal and spatial domain, there is a relevant difference between the two cases: while the returned powers from a target in the multi-frame maps pertaining an individual baseline are expected to fluctuate around the same average value, by observing a complex target such as a ship by widely separated perspectives, strong variations of the backscattered energy toward the receiver could be easily experienced. Therefore, the maps pertaining different baselines should be combined taken into accounts the different levels of reliability according to the local SNR [34]. Obviously, it is unrealistic assuming a priori knowledge of the SNR and the detector (25) is assumed as a benchmark of the achievable performance.

A practical (but suboptimum) detector can be obtained by realizing a full quadratic integration in both time and space domains, corresponding to the decision rule (25) by setting $\eta_{m}=1$ for all the baselines with a proper threshold. The final map resulting from the quadratic integrator (QI) for each tested triad $\left(f_{d r}, \phi_{t}, \dot{\phi}_{t}\right)$ is given by

$$
\begin{gathered}
R D^{Q I}\left(r, f_{u} ; f_{d r}, \phi_{t}, \dot{\phi}_{t}\right)= \\
\frac{1}{M N} \sum_{m} \sum_{n}\left|R D_{m, n}\left(r, f_{u} ; f_{d r}, \phi_{t}, \dot{\phi}_{t}\right)\right|^{2}
\end{gathered}
$$

Thanks to the energy alignment over the temporal frames and over the multiple bistatic links, the target energy builds up in the $R D^{Q I}$ map corresponding to the actual target kinematics. The time integration gain (related to the TMC) along with the space integration gain (related to the MSC), provides a SNR enhancement such that the moving target can likely compete with the disturbance contributions and therefore be detected. It has to be noted that the QI detector is a suboptimum version of the LRT. Particularly, it still realizes the optimum combination in time domain levels, while the absence of a weighting process among the different baselines is expected to provide worse results than the LRT, especially for those cases where a strong variation of the RCS over the different perspectives is experienced. The detection performance of the QI will be compared to the optimum represented by the LRT in Section IV.

Along with the detection of the target, the proposed technique aims at its simultaneous localization. The joint detection and localization of the target relies on the consideration that the time\&space integration gain is a function of the tested $\left(f_{d r}, \phi_{t}, \dot{\phi}_{t}\right)$. Therefore, the selection of the branch of the bank providing the maximum integration gain provides a direct estimate of the target kinematics. In practice, for each target detected at the generic Doppler rate $f_{d r}^{*}$ : (i) the corresponding area of interest $\mathrm{T}$ is identified in the range\&Doppler plane; (ii) the estimated values of the target DOA and DOA derivative are obtained as follows

$$
\begin{aligned}
& (\widehat{\phi}, \hat{\dot{\phi}})=\max _{\phi_{t}, \dot{\phi}_{t}} G\left(\phi_{t}, \dot{\phi}_{t}\right)=
\end{aligned}
$$

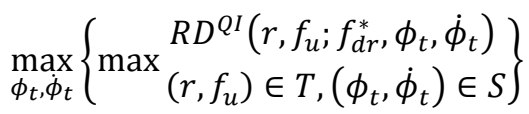


being $S$ the subset of the branches where detections arise. The estimated DOA is then combined with the range at which the target is detected (representing the target-to-receiver distance $R_{R}$ ) to retrieve its Cartesian coordinates (1).

It has to be pointed out that while the resolution is uniform in the bistatic range and Doppler plane, it is not neither in the equivalent monostatic RD plane nor in the Cartesian plane [4], [11], [24]. Consequently, the different bistatic geometries will result in differently shaped resolution cells when projected into the equivalent monostatic plane. Because of the different shapes, the intersection area among the individual resolution cells in the multistatic map can be more or less dispersed. While this is not expected to influence the detection performance (as the achieved peak power is not influenced by the resolution), it makes sense that satellites couples giving rise to very different shapes of the resolution cells are potentially able to provide an accurate DOA estimation.

Moreover, it has to be noticed that in the case of close multiple targets, the risk of merging information coming from different targets is high, carrying to the ambiguities usually referred to as ghost targets. The ghost problem is inherent to each multistatic radar system and proper strategies for its handling should be also considered. This could be a challenging task that requires specific efforts and therefore it is not treated here. Nevertheless, it is worth to mention that the large number of satellites is expected to be a key element to provide ghost rejection capability, since the target-to-target interaction giving rise to a ghost solution with a particular couple of satellites will give rise to a different ghost solution by considering different satellites; thus, with a sufficiently large formation of transmitters the ambiguities are expected to vanish.

\section{PERFORMANCE ANALYSIS}

\section{A. Detection performance}

In order to investigate the potential improvement in detection performance by exploiting the integration over the time and space domains, we refer to the $R D_{m, n}$ maps assuming ideal TMC and MSC. For sake of convenience and without loss of generality, we consider the QI operator realizing a sum (in place of a mean) of the range and Doppler cell under test (CUT) over

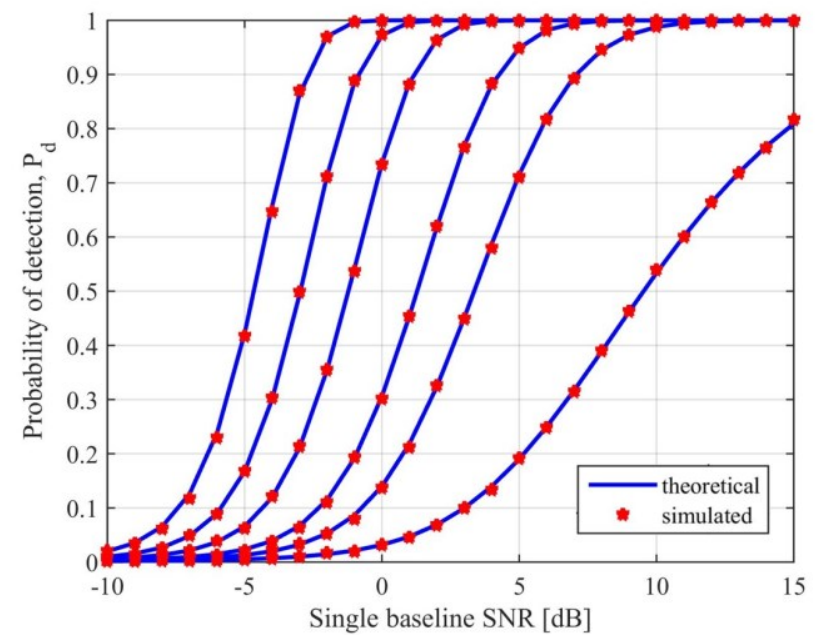

(a) the $\mathrm{N}$ frames and $\mathrm{M}$ bistatic links. We explicitly point out that with such an assumption, the QI operator coincides with the $\mathcal{L}$ detector in (25) by setting $\eta_{m}=1 \forall m$.

First, the probability of false alarm $\left(P_{f a}\right)$ for the LRT and QI operators to be used to compute the detection thresholds are presented. Then, close-form expressions for the probability of detection $\left(P_{d}\right)$ for both the detectors are provided. Full analytical details are provided in Appendix B.

Let $t_{h}^{L R T}$ and $t_{h}^{Q I}$ be the set thresholds for the LRT and QI detectors, respectively, the corresponding $P_{f a}$ are given by

$$
\begin{gathered}
P_{f a}^{L R T}=1-C_{A} \cdot \sum_{k=0}^{\infty} \delta_{A_{k}} \frac{\gamma\left(M N+k, \frac{t_{h}^{L R T} / \sigma_{w}^{2}}{\eta_{\min }}\right)}{\Gamma(M N+k)} \\
P_{f a}^{Q I}=1-\frac{\gamma\left(M N,{ }_{h}^{Q I} / \sigma_{w}^{2}\right)}{\Gamma(M N)}
\end{gathered}
$$

For a desired false alarm rate, numerical inversion of (29) and (30) provides $t_{h}^{L R T}$ and $t_{h}^{Q I}$. It should be noted that for practical purposes, (29) can be replaced with a truncated version considering the first $K^{\prime}$ elements of the infinite series according to a desired accuracy. The corresponding $P_{d}$ for the two detectors are equal to

$$
P_{d}^{L R T}=1-C_{B} \cdot \sum_{k=0}^{\infty} \delta_{B_{k}} \frac{\gamma\left(\begin{array}{c}
t_{h}^{L R T} / \sigma_{W}^{2} \\
M N+k, \frac{S^{2} R_{\min }}{2}
\end{array}\right)}{\Gamma(M N+k)}
$$

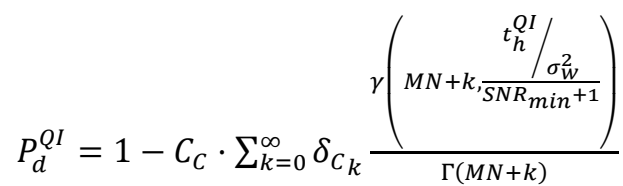

In eqs. (29)-(32), $\Gamma(\cdot)$ is the Gamma function and $\gamma(a, b)=$ $\int_{0}^{b} t^{a-1} e^{-t} d t$ is the lower incomplete Gamma function; $S N R_{\min }$ is the lowest average SNR level observed in the M

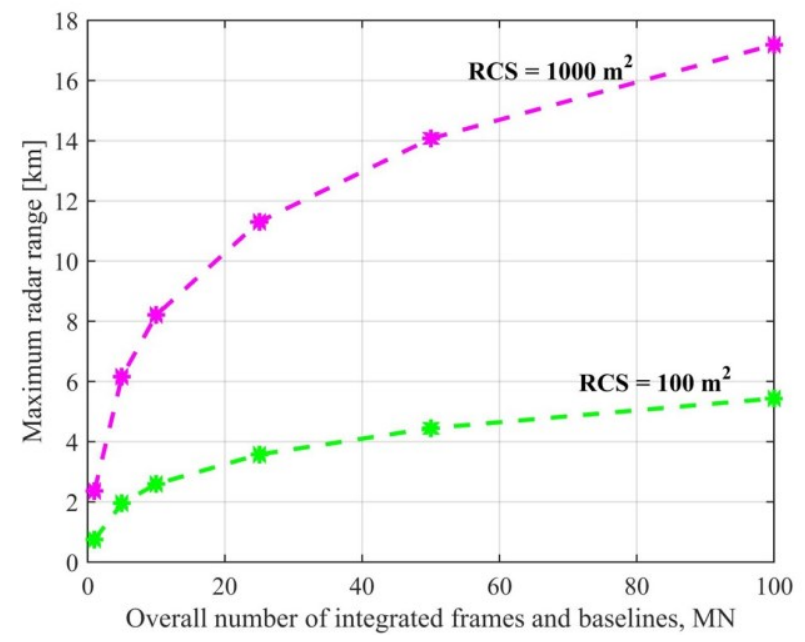

(b)

Fig. 4 Performance analysis as a function of the number of integrated frames and baselines. (a) Probability of detection. (b) Maximum radar range. 


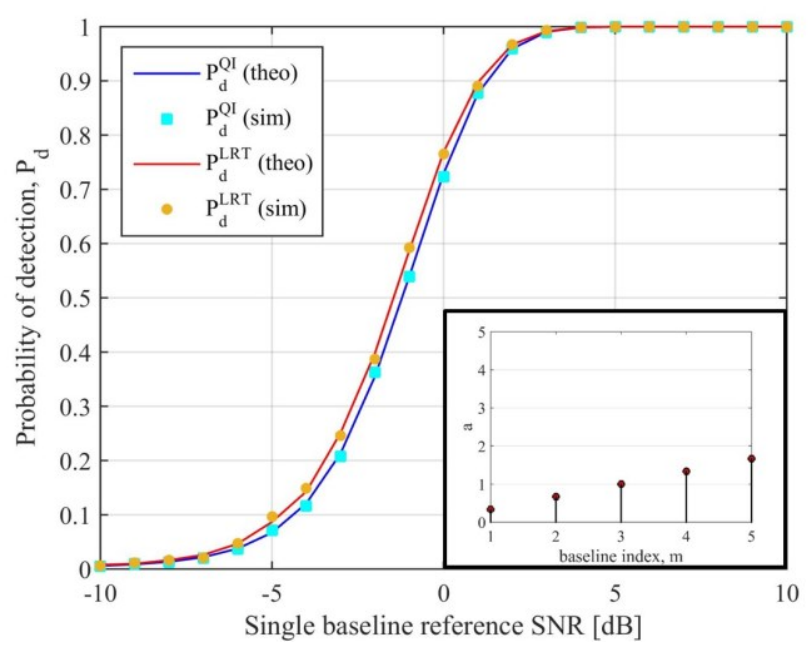

(a)

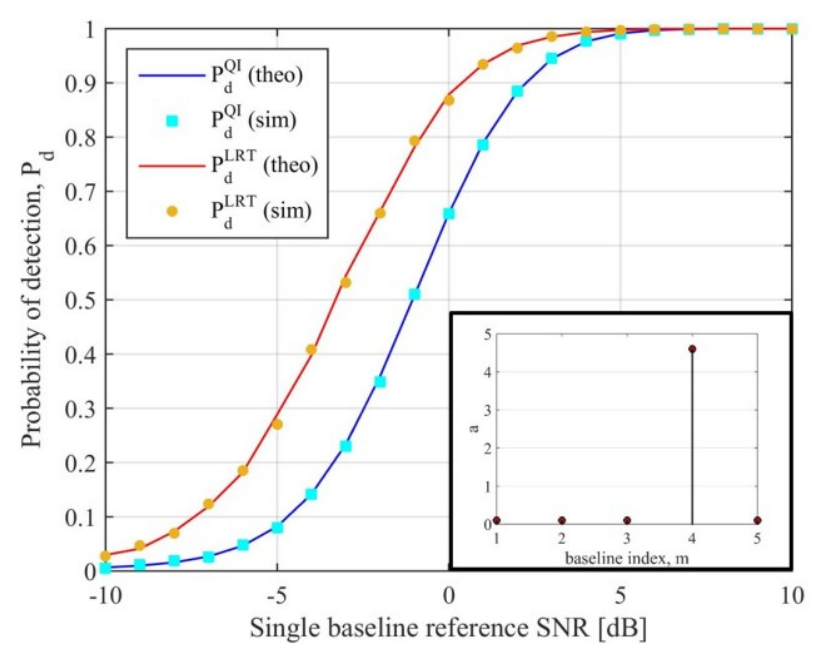

(b)

Fig. 5 LRT and QI detection performance for target RCS experiencing a smooth (a) and a strong (b) angular spread.

bistatic channel and $\eta_{\min }$ is the corresponding weight (26); parameters $C_{j}$ and $\delta_{j_{k}}(j=A, B, C)$ are reported in Appendix $\mathrm{B}$, Table B1. It is worth to notice that in the special case of equal average SNR observed at the $\mathrm{M}$ baselines, $C_{j}=1, \delta_{j_{0}}=1$ and $\delta_{j_{k>0}}=0$, therefore (29), (31) and (32) reduces at common expressions of $P_{f a}$ and $P_{d}$ for gamma variates.

In a first case study, we assume that the target power fluctuates frame-to-frame according to a Swerling I model with the same average value among the different links (i.e., $\sigma_{m}^{2}=$ $\left.\sigma^{2} \forall m\right)$. We explicitly point out that in this particular case both LRT and QI detectors realize an unweighted sum of the data, thus their performance coincide. Fig. 4a shows the probability of detection as a function of the SNR pertaining the individual baseline (average SNR observed in a single frame). Threshold has been set to achieve a false alarm ratio of 0.001 . Full lines represent the theoretical $P_{d}$, while red markers show the corresponding result achieved by Monte Carlo simulations (10000 independent trials for each tested SNR). From the figure, we can observe as the detection performance progressively enhances by increasing the number of integrated maps, wheatear they are multi-frame maps pertaining a single satellite, single frame maps pertaining multiple satellites, or multi-frame and multi-satellite maps. For example, only highRCS targets at relatively short ranges able to provide SNR greater than $15 \mathrm{~dB}$ could be detected with $P_{d}>0.9$ in the map achieved over a short time (namely, single frame) exploiting an individual satellite. The non-coherent integration of $\mathrm{N}=10$ motion compensated maps allows to achieve the same detection performance for considerably lower RCS (and/or at higher ranges) targets, with a required single map average SNR equal to $4.2 \mathrm{~dB}$. By further considering the integration of multiple baselines we can reduce the required SNR at $-1 \mathrm{~dB}$ and $-2.5 \mathrm{~dB}$ considering $\mathrm{M}=5$ and $\mathrm{M}=10$ satellites, respectively. Therefore, a large integration gain is made available by properly exploiting the multiple satellites simultaneously illuminating the scene, which enables the detection of targets not observable with the individual bistatic geometry, even exploiting a long integration time. Moreover, the improvement of the performance enabled by multiple satellites can be also used to reduce the number of integrated frames. It should be pointed out that the temporal integration gain provided by the TMC could be affected by not negligible target maneuvers, resulting in higher order terms of the Doppler migration. By trading part of the integrated frames with a greater number of satellites, the robustness of the TMC can be improved making

TABLE I SIMULATED SCENARIO - SATELLITES PARAMETERS

\begin{tabular}{|c|c|c|c|}
\hline Parameter & & Unit & Value \\
\hline \multirow{5}{*}{ Satellite 1} & \multirow{5}{*}{$\begin{array}{l}\text { Number } \\
\text { Azimuth angle (at reference } \\
\text { instant) } \phi_{1} \\
\text { Azimuth angle rate } \dot{\phi}_{1} \\
\text { Elevation angle (at reference } \\
\text { instant) } \theta_{1} \\
\text { Elevation angle rate } \dot{\theta}_{1}\end{array}$} & - & GSAT0204 \\
\hline & & deg & -110.70 \\
\hline & & $\operatorname{deg} / \mathrm{s}$ & 0.0057 \\
\hline & & deg & 53.57 \\
\hline & & $\mathrm{deg} / \mathrm{s}$ & 0.0056 \\
\hline \multirow{5}{*}{ Satellite 2} & \multirow{5}{*}{$\begin{array}{l}\text { Number } \\
\text { Azimuth angle (at reference } \\
\text { instant) } \phi_{2} \\
\text { Azimuth angle rate } \dot{\phi}_{2} \\
\text { Elevation angle (at reference } \\
\text { instant) } \theta_{2} \\
\text { Elevation angle rate } \dot{\theta}_{2}\end{array}$} & - & $\overline{\text { GSAT0206 }}$ \\
\hline & & deg & -81.81 \\
\hline & & $\operatorname{deg} / \mathrm{s}$ & -0.0106 \\
\hline & & deg & 53.74 \\
\hline & & $\operatorname{deg} / \mathrm{s}$ & 0.0006 \\
\hline \multirow{5}{*}{ Satellite 3} & \multirow{5}{*}{$\begin{array}{l}\text { Number } \\
\text { Azimuth angle (at reference } \\
\text { instant) } \phi_{3} \\
\text { Azimuth angle rate } \dot{\phi}_{3} \\
\text { Elevation angle (at reference } \\
\text { instant) } \theta_{3} \\
\text { Elevation angle rate } \dot{\theta}_{3}\end{array}$} & - & GSAT0210 \\
\hline & & deg & -25.72 \\
\hline & & $\operatorname{deg} / \mathrm{s}$ & -0.0053 \\
\hline & & deg & 9.16 \\
\hline & & $\operatorname{deg} / \mathrm{s}$ & -0.0002 \\
\hline \multirow{5}{*}{ Satellite 4} & \multirow{5}{*}{$\begin{array}{l}\text { Number } \\
\text { Azimuth angle (at reference } \\
\text { instant) } \phi_{4} \\
\text { Azimuth angle rate } \dot{\phi}_{4} \\
\text { Elevation angle (at reference } \\
\text { instant) } \theta_{4} \\
\text { Elevation angle rate } \dot{\theta}_{4}\end{array}$} & - & GSAT0214 \\
\hline & & deg & 54.01 \\
\hline & & $\operatorname{deg} / \mathrm{s}$ & -0.0036 \\
\hline & & deg & 35.92 \\
\hline & & $\operatorname{deg} / \mathrm{s}$ & -0.0045 \\
\hline
\end{tabular}

it less sensitive to possible signal model mismatches due to more complex target motions.

Moreover, Fig. $4 \mathrm{~b}$ shows the corresponding maximum radar range achievable for targets having average $\mathrm{RCS}=100 \mathrm{~m}^{2}$ and $1000 \mathrm{~m}^{2}$ as a function of the overall number of integrated 


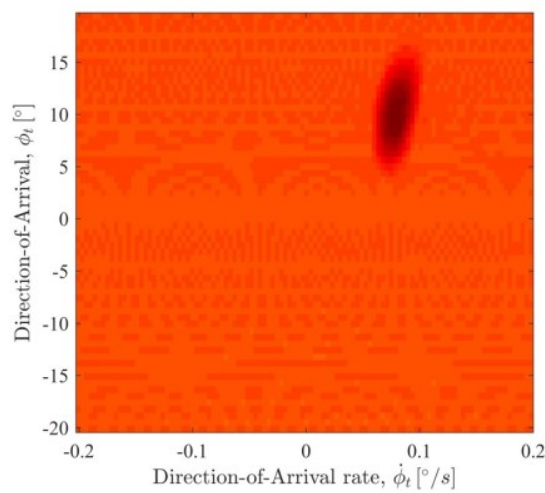

(a)

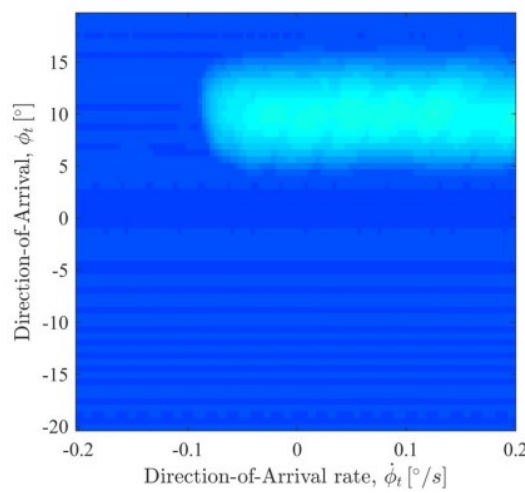

(b)

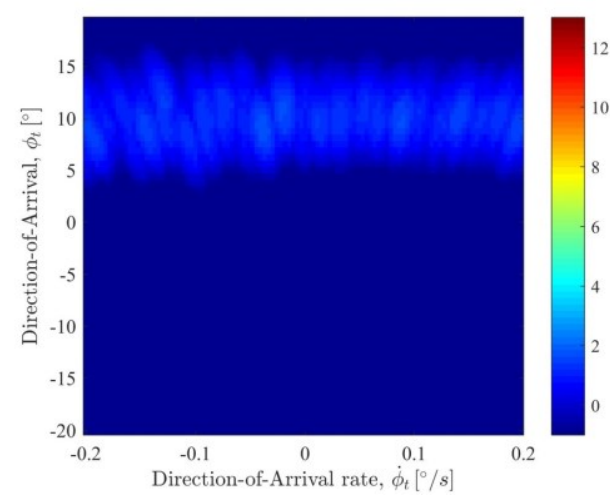

(c)

Fig. 6 Integration gain (dB scale) for (a) $f_{d r}=-0.011 \mathrm{~Hz} / \mathrm{s}$ (actual value), (b) $f_{d r}=-0.100 \mathrm{~Hz} / \mathrm{s}$ and (c) $f_{d r}=-0.333 \mathrm{~Hz} / \mathrm{s}$.

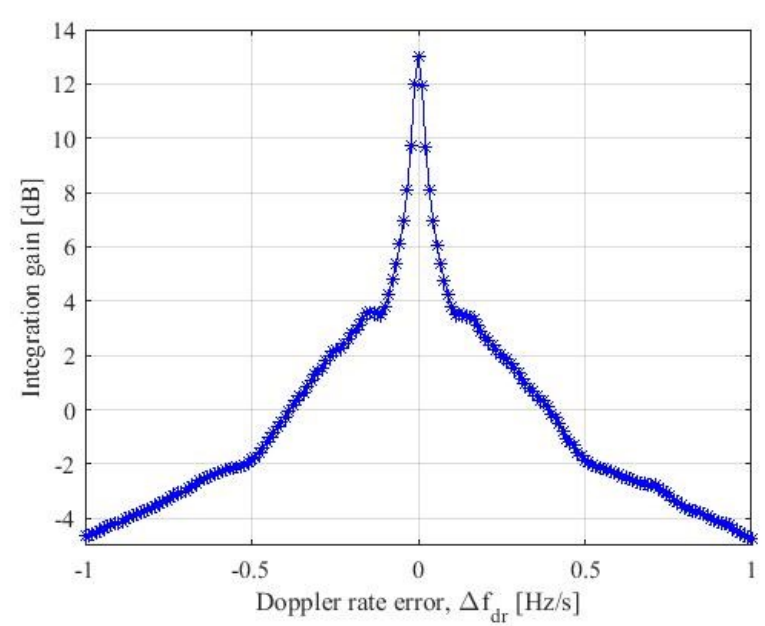

Fig. 7 Integration gain as a function of the Doppler rate error for the actual DOA and DOA rate.

frames and baselines for desired $P_{f a}=10^{-3}$ and $P_{d}=75 \%$. To evaluate the link budget, the flux power density on the sea level has been set equal to $3 \times 10^{-14} \mathrm{~W} / \mathrm{m}^{2}$, receiver surveillance antenna gain equal to $15 \mathrm{~dB}$ with noise figure 1.5 $\mathrm{dB} ; 2 \mathrm{~dB}$ of system losses have been also considered and frame length has been set equal to $3 \mathrm{~s}$. The results in the figure clearly show the enhanced coverage capability enabled by the time and space integration, potentially allowing the detection of ship targets with $\mathrm{RCS} \approx 30 \mathrm{dBm}^{2}$ (that could for example correspond to medium coasters) up to $10-20 \mathrm{~km}$.

As already underlined, the different illumination angles due to the multiple transmitters can result in large variations of the target RCS. The detection performance of the QI operator is here tested and compared to the ideal performance achieved by the LRT for a few scenarios considering different variation of the average RCS observed by the multiple bistatic geometries. $\mathrm{N}=5$ frames and $\mathrm{M}=5$ satellites are considered. The average RCS observed by the $m$ th baseline during the integration time is set according to the rule $\sigma_{m}=a_{m} \bar{\sigma}$, where $a_{m}$ are weighting coefficients such that $\sum_{m} a_{m}=M$. Therefore, the average target power observed in each frame by the multistatic system is $M \bar{\sigma}$. Fig. 5 shows the theoretical and simulated probability of detection (for $P_{f a}$ set to 0.001 ) for the LRT and QI detectors as a function of the single baseline reference SNR (i.e., $\bar{\sigma} / \sigma_{w}$ ) for different spread of the target RCS over the baselines. The weight coefficients defining the entity of the RCS variations are shown in the boxes in the bottom of the figures. Fig. 5a shows the case of a smooth spreading ( $a$ coefficients are $0.33,0.67,1$, $1.33,1.67)$ and as it is apparent LRT and QI detectors obtain very similar performance. In Fig. 5b a larger spreading has been considered (a coefficients are $0.1,0.1,0.1,4.96,0.1$ ). In particular, this scenario would represent the situation in which the energy backscattered from the target is much stronger for some particular bistatic angle than other perspectives. Obviously, in such a case the main diversity gain consists in the higher chances to intercept the favorable bistatic angles. In terms of detector performance, we can observe that LRT provides some improvement moving from the smooth to the strong RCS angular spread while the QI experiences some losses. However, these variations are rather limited, and the QI loss with respect the optimum performance are not greater than about $2 \mathrm{~dB}$, thus proving the robustness of the QI operator against the RCS angular spread.

\section{B. Localization capabilities}

The possibility to localize the detected target in the local reference system is here tested against synthetic data. Four satellites of Galileo constellation are considered as transmitters of opportunity, exploiting signals transmitted in the E5a band. The satellites trajectories were obtained from a GNSS satellite tracking website [35] and the main parameters are listed in Table I. The target is moving in the surveyed area being at the reference time instant (centre of the overall dwell time) at the distance of $1500 \mathrm{~m}$ from the receiver with a direction of arrival $\phi_{t}=10^{\circ}$. The target velocity is $7 \mathrm{kn}$ with heading $45^{\circ}$, corresponding to a DOA rate $\dot{\phi}_{t}=0.079^{\circ} / \mathrm{s}$. The entire dwell time is set to be 30 seconds, segmented in $\mathrm{N}=10$ frames of duration $\mathrm{T}_{\mathrm{f}}=3 \mathrm{~s}$.

To show clearly the localization capability of the proposed technique, the analysis is provided in noise-free conditions accounting for a target with constant RCS. Fig. 6 shows the function $G$ in (28) obtained by considering two transmitters (sat. 1 and sat. 2 in Table I) for three different values of the Doppler rate; in particular, Fig. 6a corresponds to the actual value of the Doppler rate $f_{d r}=-0.011 \mathrm{~Hz} / \mathrm{s}$, while Fig. $6 \mathrm{~b}$ and c pertain wrong $f_{d r}$ values $(-0.100 \mathrm{~Hz} / \mathrm{s}$ and $-0.333 \mathrm{~Hz} / \mathrm{s}$, respectively). In the figures, $0 \mathrm{~dB}$ denotes the target power evaluated at the individual $\mathrm{RD}$ map level (i.e. single $\mathrm{CPI}, \mathrm{N}=1$, and single 


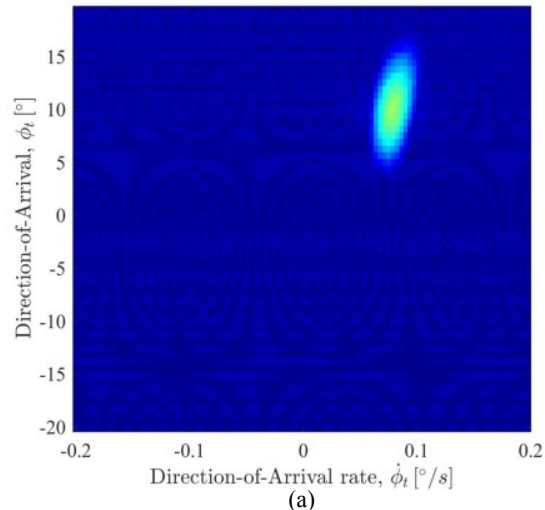

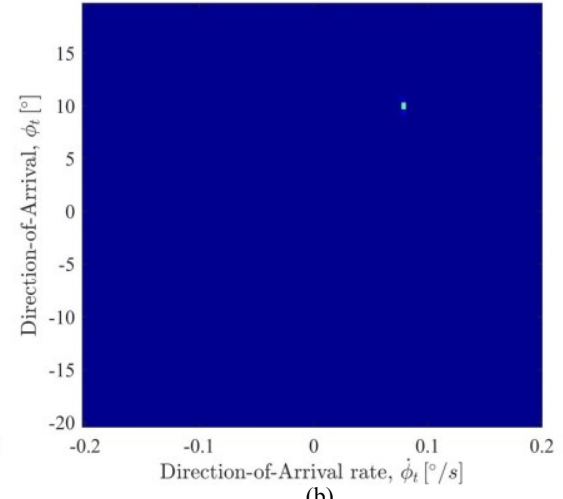

(b)

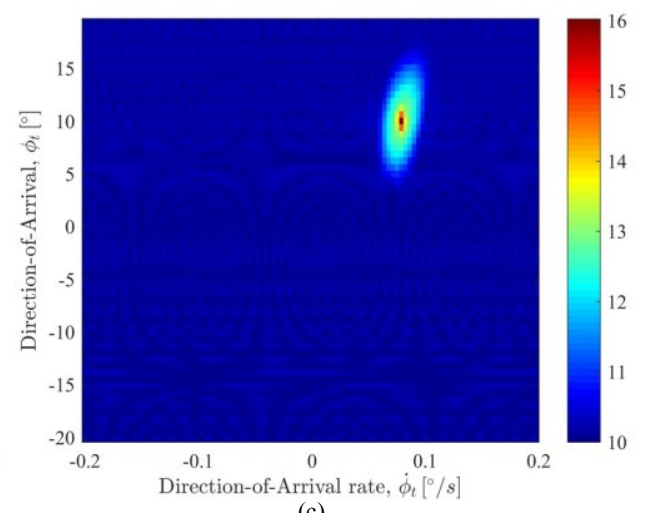

(c)

Fig. 8 Integration gain (dB scale) considering (a) $\mathrm{M}=2$ (sat.1 and sat. 2, low spatial diversity), (b) $\mathrm{M}=2$ (sat. 3 and sat. 4 , large spatial diversity), (c) $\mathrm{M}=$

4.

bistatic link, $\mathrm{M}=1$ ). Therefore, a maximum gain of $13 \mathrm{~dB}$ can be obtained when all the frames and baselines contributions are correctly aligned. It can be observed that such a value is reached in the slice pertaining the actual Doppler rate in correspondence of the actual angular values $\left(\phi_{t}, \dot{\phi}_{t}\right)$. Therefore, a direct estimate of the target DOA can be obtained and combined with

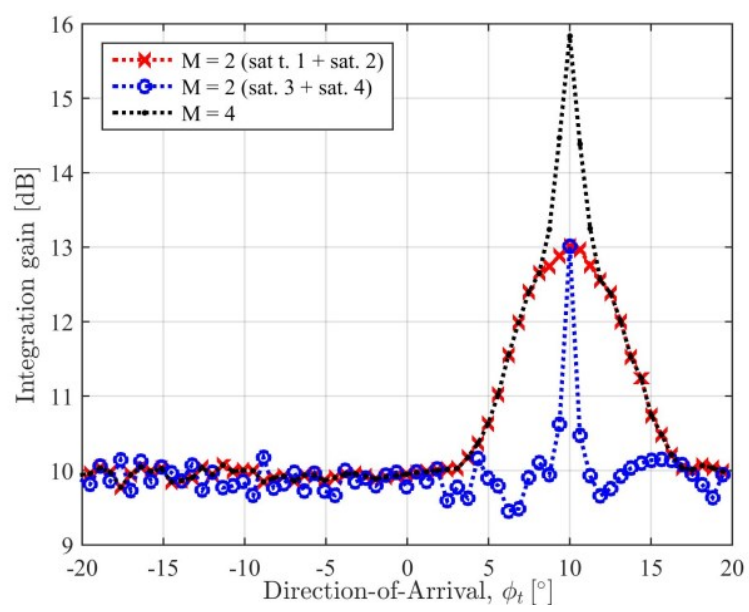

Fig. 9 Integration gain as a function of the tested DOA for the actual Doppler rate and DOA rate.

the retrieved monostatic range to enable target localization. Comparing the three figures, it is interesting to note the different levels of background. Indeed, it represents the integration gain resulting from the multi-frame combination only: therefore, the highest value is obtained for the actual Doppler rate, providing best TMC performance. When the TMC is performed with wrong Doppler rate values, both Doppler migration inside the frame and range and Doppler migration among the frames are not correctly compensated. Due to the target energy spread over multiple cells, the multistatic compensation procedure cannot relocate the target contributions around a single position and therefore a spread of the integration gain over multiple $\left(\phi_{t}, \dot{\phi}_{t}\right)$ couples occurs. To better highlight the effect of the Doppler rate on the achieved integration gain, Fig. 7 shows the integration gain variation as a function of the Doppler rate offset $\Delta f_{d r}$ between the tested and the actual Doppler rate by fixing the actual values of $\phi_{t}$ and $\dot{\phi}_{t}$. As it is apparent, the maximum is obtained for $\Delta f_{d r}=0$ $\mathrm{Hz} / \mathrm{s}$.

Likewise the multilateration approach, it is expected that the capability of the proposed technique to localize the target depends on the degree of spatial diversity offered by the selected transmitters. If a single couple of transmitters is selected, it makes sense that the higher is the difference in bistatic iso-range contours, the higher is the accuracy of the estimated DOA. Fig. 8 shows the function $G$ in (28) achieved under three different conditions. Fig. 8a and b show $G$ when the couple (sat. 1, sat. 2) and (sat. 3, sat. 4) have been selected, respectively. It could be shown that sat. 3 and sat. 4 have a larger difference in bistatic iso-ranges than sat. 1 and sat. 2 . Consequently, the MSC is able to better concentrate the target energy in a smaller region in the $\left(\phi_{t}, \dot{\phi}_{t}\right)$ plane, as it can be observed comparing the two images, likely implying a greater accuracy in the DOA estimation. Fig. 8c shows $G$ when all the satellites have been selected, where the higher integration gain due to the greater number of considered baselines is easily observed. Therefore, increasing the number of considered satellites can improve not only the signal to background conditions but also the quality of the localization of the detected target thanks to the higher energy concentration in the $\left(\phi_{t}, \dot{\phi}_{t}\right)$ plane. The enhancement of the capability of the proposed approach at providing more accurate DOA estimates by considering more satellites with higher spatial diversity can be better observed by looking at Fig. 9 showing a cut along the tested DOA dimension around the actual $\left(\phi_{t}, \dot{\phi}_{t}\right)$ couple.

\section{EXPERIMENTAL RESULTS}

An experimental campaign was conducted inside the H2020 SpyGLASS project, [38]. Proof-of-concepts experiments were conducted in two different scenarios: river shipping and port operations. Galileo satellites have been exploited and more in detail the pilot signals transmitted on the frequency band E5a (carrier frequency: $1176.45 \mathrm{MHz}$ ). The passive receiver was equipped with two channels to collect direct and reflected signals. Particularly, the reference channel used a low-gain antenna while the surveillance channel used a higher gain antenna pointed toward the surveyed area. In order to have a reference ground truth for comparison, the following analysis focuses on targets of opportunity equipped with Automatic 


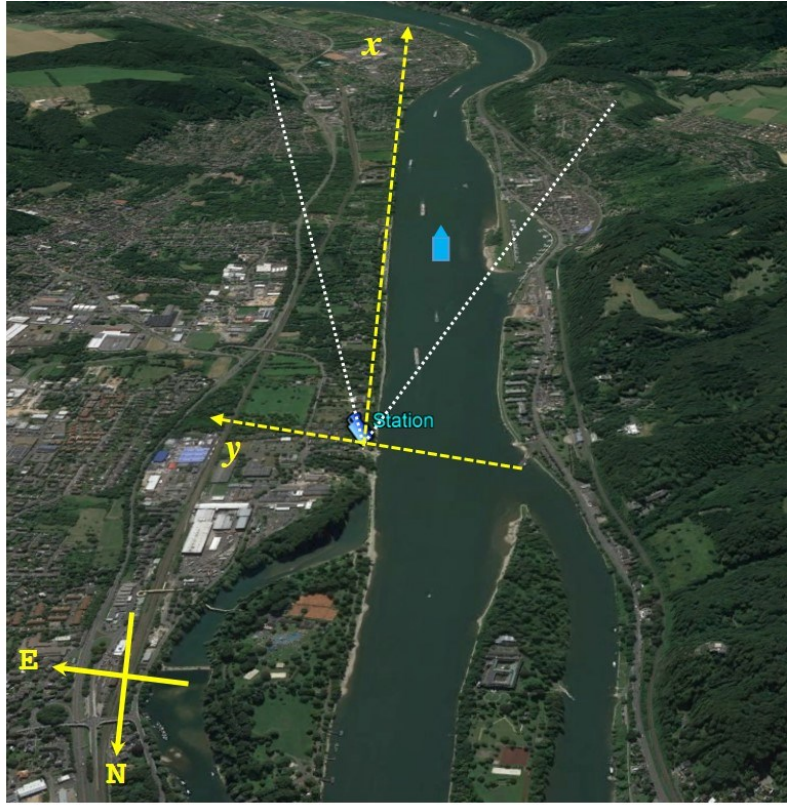

(a)

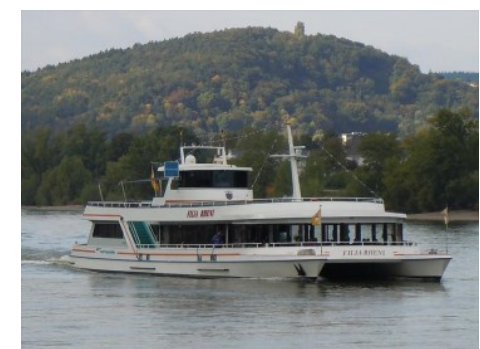

(b)

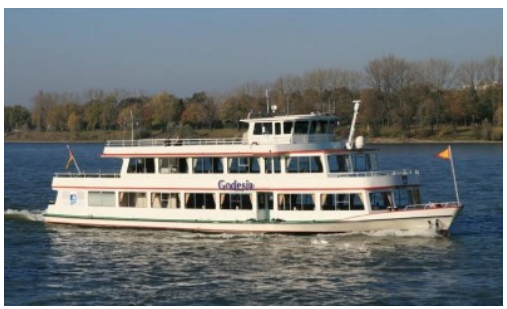

(c)

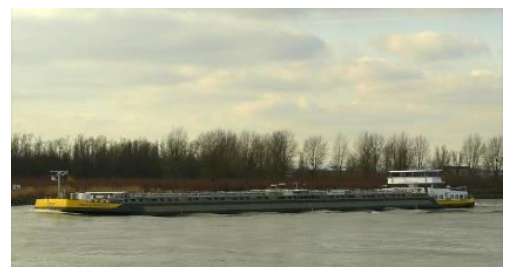

(d)

Fig. 10 River navigation experimental trials. (a) Acquisition geometry.

(b) 'Filia Rheni' optical photograph [36]. (c) 'Godesia' optical photograph [36]. (d) 'Orange Nassau II' optical photograph [36].

Identification System (AIS) whose messages were recorded during all the acquisitions.

\section{A. Scenario 1: River shipping}

In the first experimental campaign the receiver was located on the Rhine riverside near Bonn, observing the vessel traffic mainly composed by barges, car ferries and cruise ships. Fig. 10a shows the geometry of the acquisition, during which the surveillance antenna was pointed toward South. In a two mins
TABLE II SHIPPING RIVER EXPERIMENTAL CAMPAIGN - TRACKED SATELLITES

\begin{tabular}{llll}
\hline Parameter & & Unit & Value \\
\hline \multirow{4}{*}{ Satellite 1} & Number & - & GSAT0210 \\
& Ranging code & - & E01 (E5a-Q) \\
& Azimuth angle & $\operatorname{deg}$ & $164.38 \sim 163.75$ \\
& Elevation angle & $\operatorname{deg}$ & $9.17 \sim 9.14$ \\
\hline \multirow{5}{*}{ Satellite 2 } & Number & - & GSAT0214 \\
& Ranging code & - & E05 (E5a-Q) \\
& Azimuth angle & $\operatorname{deg}$ & $-115.90 \sim-116.32$ \\
& Elevation angle & $\operatorname{deg}$ & $36.02 \sim 35.48$ \\
\hline
\end{tabular}

long acquisition, the two passenger ferries 'Filia Rheni' (Fig. $10 \mathrm{~b})$ and 'Godesia' (Fig. 10c), with size (length overall $\times$ breadth extreme) equal to $42 \mathrm{~m} \times 11 \mathrm{~m}$ and $38.6 \mathrm{~m} \times 8.6 \mathrm{~m}$, respectively, were moving away from the receiver. Moreover, the barge 'Orange Nassau II' (Fig. 10d) of size $110 \mathrm{~m} \times 13.5 \mathrm{~m}$ was moving toward the receiver going out of the surveyed area at the beginning of the acquisition. Two Galileo satellites were correctly tracked during the acquisition, whose parameters are listed in Table II.

Fig. 11 shows the RD maps pertaining a single frame for the two acquired satellites. The $0 \mathrm{~dB}$ denotes the mean background disturbance level. As it is apparent, in both the images we cannot observe any bright spots that can be associated with a target. The short CPI considered did not suffice to bring the target out from the disturbance background, highlighting the necessity to achieve a further integration gain by exploiting longer integration times and multiple baselines.

Fig. 12 shows the bistatic multi-frame RD maps obtained by the non-coherent integration of the first $\mathrm{N}=10$ frames by applying the TMC (and skipping in this case the MSC) and selecting the branch of the Doppler rate bank providing the highest SNR in the area where the ferries are located. Particularly, the highest SNR is reached on the same branch for the two ferries: this is in agreement with the recorded AIS information stating that the Doppler rate difference between the two targets was lower than the step in (15). In Fig. 12a pertaining sat. 1, three bright spots are well visible. Comparing with the AIS information, these correspond to 'Filia Rheni', 'Godesia' and 'Orange Nassau II'. In Fig. 12b, pertaining sat. 2 , only one clear intensity peak can be observed for negative Doppler values, corresponding to 'Filia Rheni', while 'Godesia' seems being not detectable in this bistatic map, likely due to a lower bistatic RCS resulting from sat. 2 perspective. This can be regarded as an experimental evidence of the strong dependence of the target RCS on the bistatic angle. Moreover, in Fig. 12 further bright spots are visible in the positive Doppler region, around $(340 \mathrm{~m}, 24 \mathrm{~Hz})$ for sat. 1 and $(250 \mathrm{~m}, 32 \mathrm{~Hz})$ for sat. 2. It could be shown by the comparison with the AIS that these correspond to 'Orange Nassau II'. It is worth to point out how these spots are not well focused. Such a blurring effect can be explained recalling that these maps have been obtained by a TMC procedure maximizing the return for the two ferries. As the different kinematic parameters of 'Orange Nassau II', a different Doppler rate should be considered for correctly focusing its energy over the long dwell.

In the long-time bistatic RD maps in Fig. 12, 'Filia Rheni' energy was concentrated around the positions (1314 m, -18.9 $\mathrm{Hz})$ and $(1129 \mathrm{~m},-14.3 \mathrm{~Hz})$ for sat.1 and sat. 2, respectively. As 


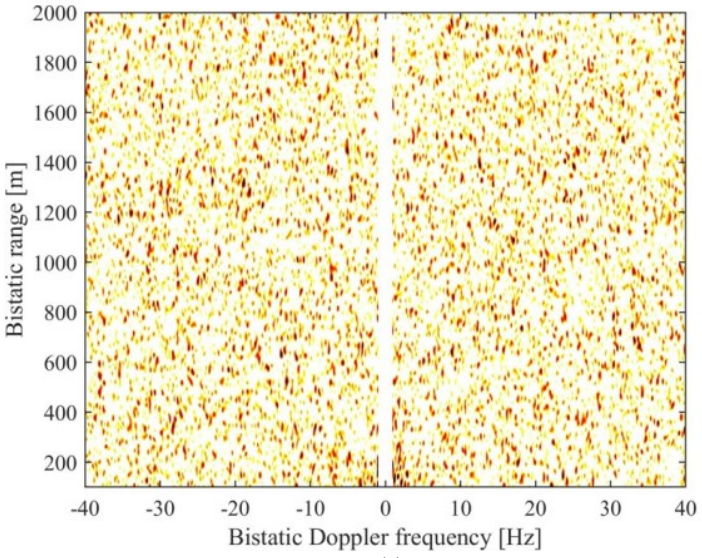

(a)

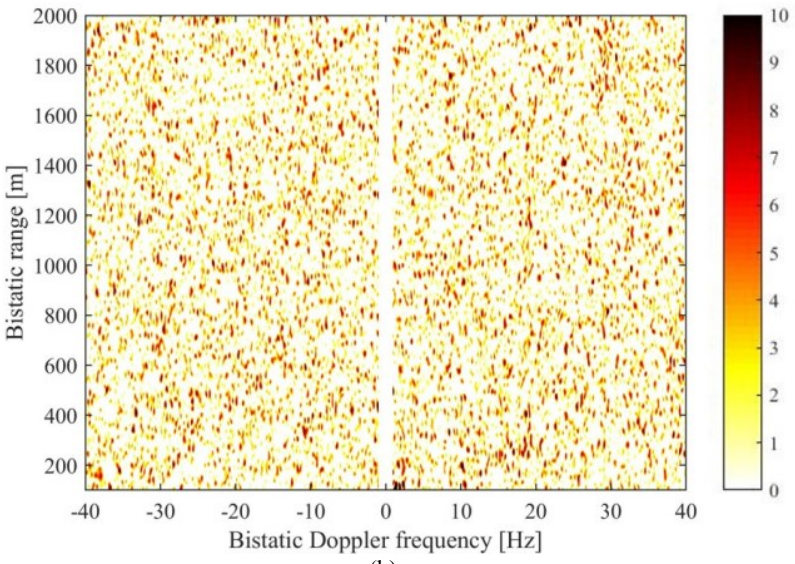

(b)

Fig. 11 Bistatic single frame RD maps. (a) sat. 1. (b) sat. 2.

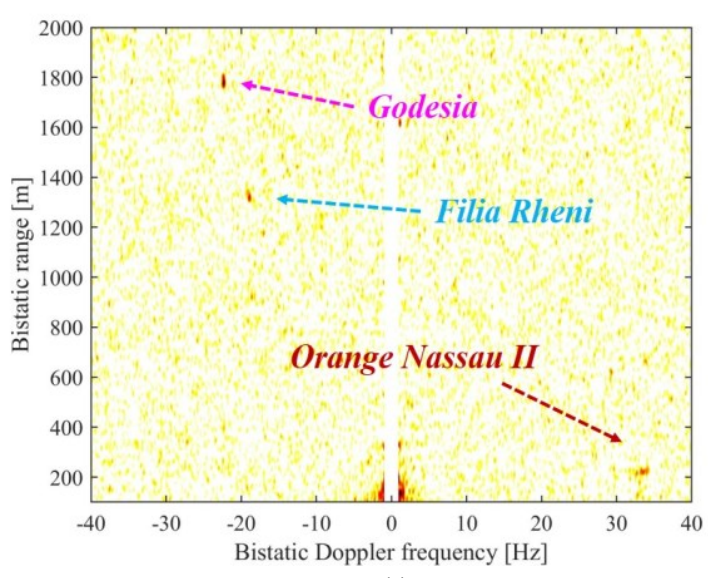

(a)

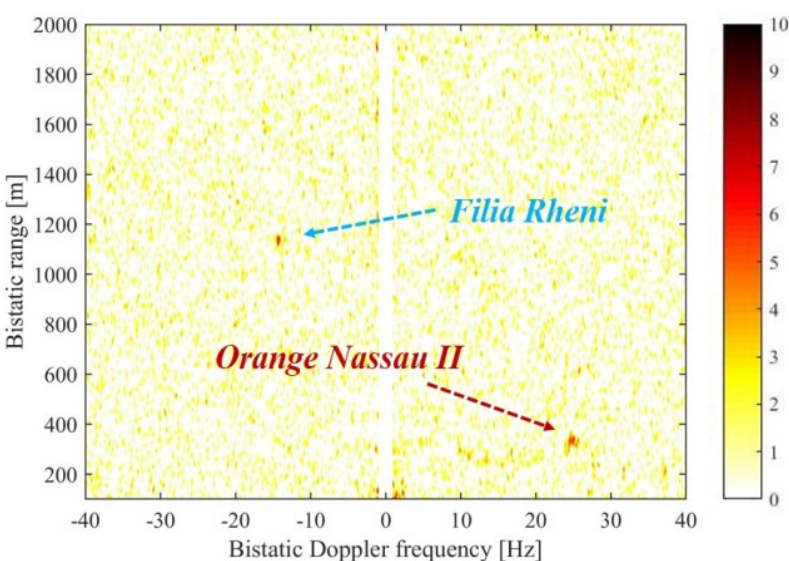

(b)

Fig. 12 Bistatic multi-frame RD maps. (a) sat. 1. (b) sat. 2.

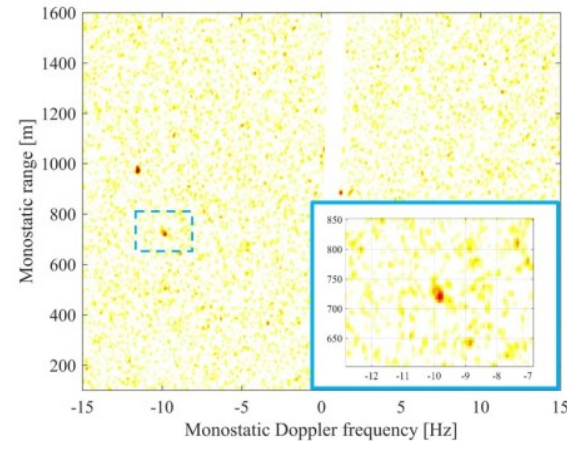

(a)

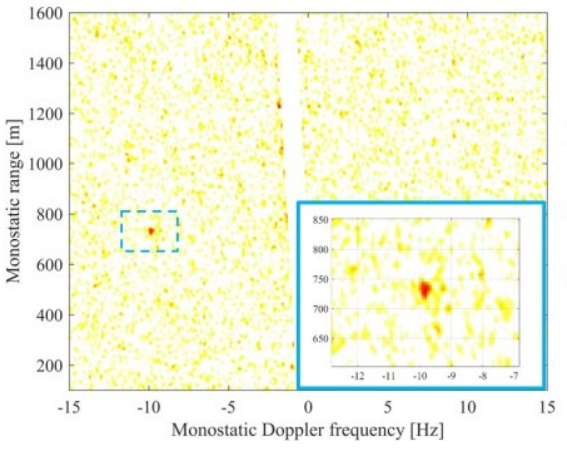

(b)

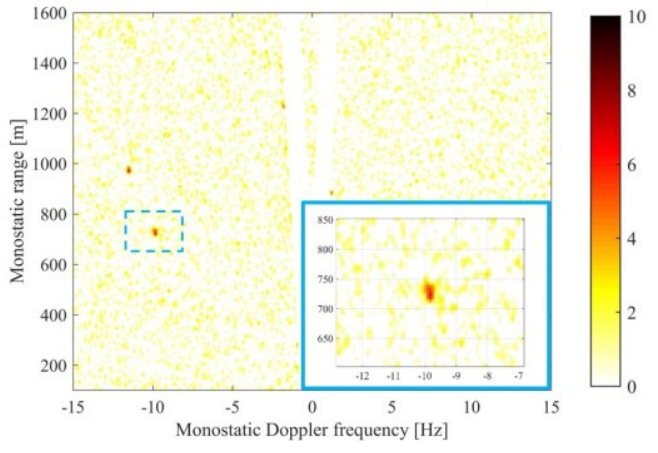

(c)

Fig. 13 Space-aligned long-time bistatic and multistatic RD maps corresponding to 'Filia Rheni' actual kinematics. (a) long-time monostatic map sat. 1. (b) long-time monostatic map sat. 2. (c) long-time multistatic map.

a consequence, the two maps cannot be directly summed to improve the SNR. The whole procedure comprising both TMC and MSC has been then applied to the data for the Doppler rate maximizing the SNR. Fig. 13 shows the resulting RD maps when the 'Filia Rheni' angular values provided by (28) have been considered. In the figure, the axes represent the range and Doppler pertaining the target-to-receiver distance and where 0 $\mathrm{dB}$ denotes again the mean disturbance level. In particular, Fig. $13 \mathrm{a}$ and $\mathrm{b}$ show the long-time monostatic maps. The blue boxes highlight the area where the target energy is expected and we can observe that after the MSC it locates around the same position $(725 \mathrm{~m},-9.8 \mathrm{~Hz})$. Therefore, SNR improvement can be obtained in the corresponding long-time multistatic RD map, shown in Fig. 13c. We explicitly point out that the further spot visible in Fig. 13a is due to 'Godesia', whose energy is combined with resolution cells containing background values only resulting in the spot visible in Fig. 13c. Comparing the three images, a lower level of the disturbance fluctuations can be appreciated in the multistatic image, while the 'Filia Rheni' power is the mean of the peak powers in the individual longtime bistatic images. Even though the exploitation of a single couple of bistatic links does not allow to considerably enhance 


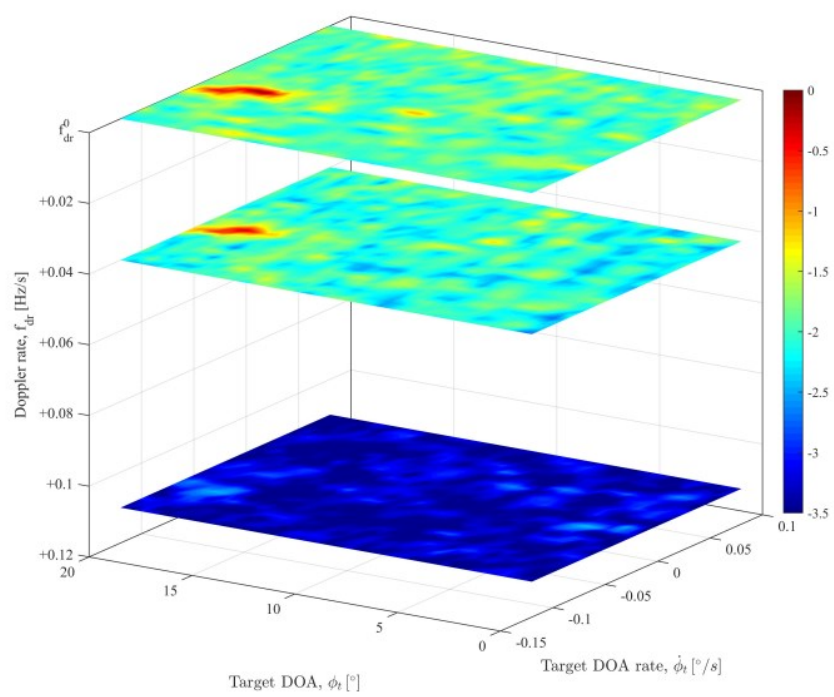

Fig. 14 'Filia Rheni' integration gain cost function $G\left(f_{d r}, \phi_{t}, \dot{\phi}_{t}\right)$.

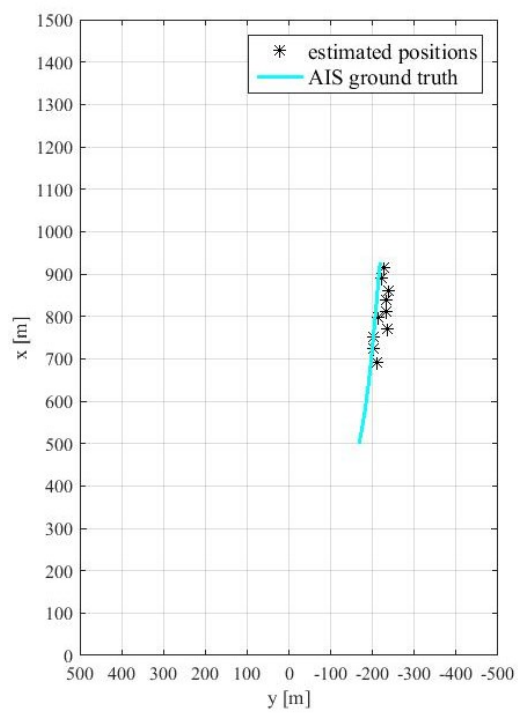

(a)

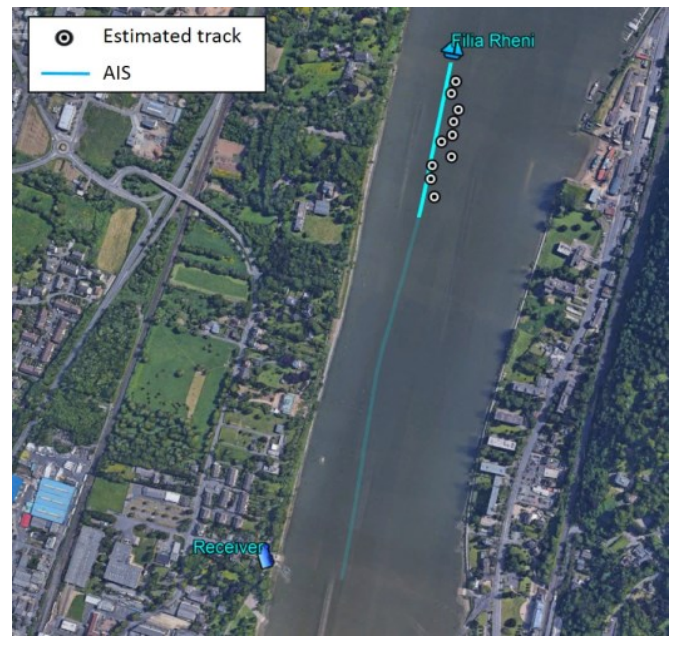

(b)

Fig. 15 'Filia Rheni’ track. (a) Cartesian plane. (b) Geographical plane. the detection performance of the system, the provided results shows the effectiveness of the method: by considering a larger number of transmitters, even considering multiple fully lunched constellations, the SNR improvement could suffice to enable detections of lower observable targets, such as 'Godesia'.

As well as the improvement of the detection performance, the proposed method enables the joint localization of the target by looking for the tested angular values providing the maximum gain. Particularly, Fig. 14 shows the function $G$ in (28) (in logscale) for three different values of the tested Doppler rate when the first $30 \mathrm{~s}$ of the acquisition have been selected $\left(T_{\mathrm{f}}=3 \mathrm{~s}, \mathrm{~N}\right.$ $=10$ ). The figures have been normalized to the maximum value, which is found in the slice on the top corresponding to the nominal Doppler rate $f_{d r}^{0}$. Moving to the bottom slices (increasing offset with respect to $f_{d r}^{0}$ ), we can observe a lower mean level due to the worse temporal integration gain provided by the TMC according to wrong values of the Doppler rate. At the same time, the spatial integration gain provided by the MSC reaches its maximum in correspondence of a specific couple $\left(\phi_{t}, \dot{\phi}_{t}\right)$ in the slice pertaining $f_{d r}^{0}$. Such couple is retained as the estimated values of the target DOA and its rate of change. From the estimated monostatic range and DOA the target Cartesian coordinates are obtained. Fig. 15 shows the estimated instantaneous target positions obtained by means of this procedure applied over consecutive integration windows with an interval of $10 \mathrm{~s}$ between two consecutive windows. In particular, Fig. 15a shows the estimated track in the $(x, y)$ plane, whereas Fig. $15 \mathrm{~b}$ reports the same information in the geographical plane. As it is apparent, a good agreement between the estimated track and the AIS ground truth has been obtained, with a Root Mean Square Error (RMSE) between the two trajectories of about $20 \mathrm{~m}$, namely even lower than the target maximum extension.

\section{B. Scenario 2: Port operations}

The second acquisition campaign was conducted in the premises of the Marghera Port, Italy. The receiver was located at the entrance of the port area, acquiring the signals scattered by the commercial vessels sailing in the port entry/exit channel, see Fig. 16a. Marghera Port is a commercial harbour and therefore the large majority of passing vessels were massive container ships and tankers. Fig. 16b shows an optical photograph of one of such targets, 'Fairpartner' $(143.1 \mathrm{~m} \times$ $26.6 \mathrm{~m}$ ), which was observed entering in the port terminal in a 3 mins long acquisition during which four Galileo satellites were correctly tracked. Table III lists the parameters of the tracked satellites.

Due to the large size of the considered target of opportunity, it is expected that even a conventional short time MTI approach can suffice to detect the target. Fig. 17 shows the single frame bistatic RD maps $\left(T_{f}=3 \mathrm{~s}\right)$ for the different baselines, where the target position provided by the AIS data is highlighted by the black dotted circle. It can be seen that with the sat. 1, a large SNR is achieved, with a peak power of about $30 \mathrm{~dB}$ above the mean disturbance level. Nevertheless, by considering the remainder satellites, much lower peak powers are obtained. The target is barely visible with sat. 2 and sat. 3 , while it is completely buried in the disturbance background with sat. 4 . The different SNR levels observed at the different bistatic 


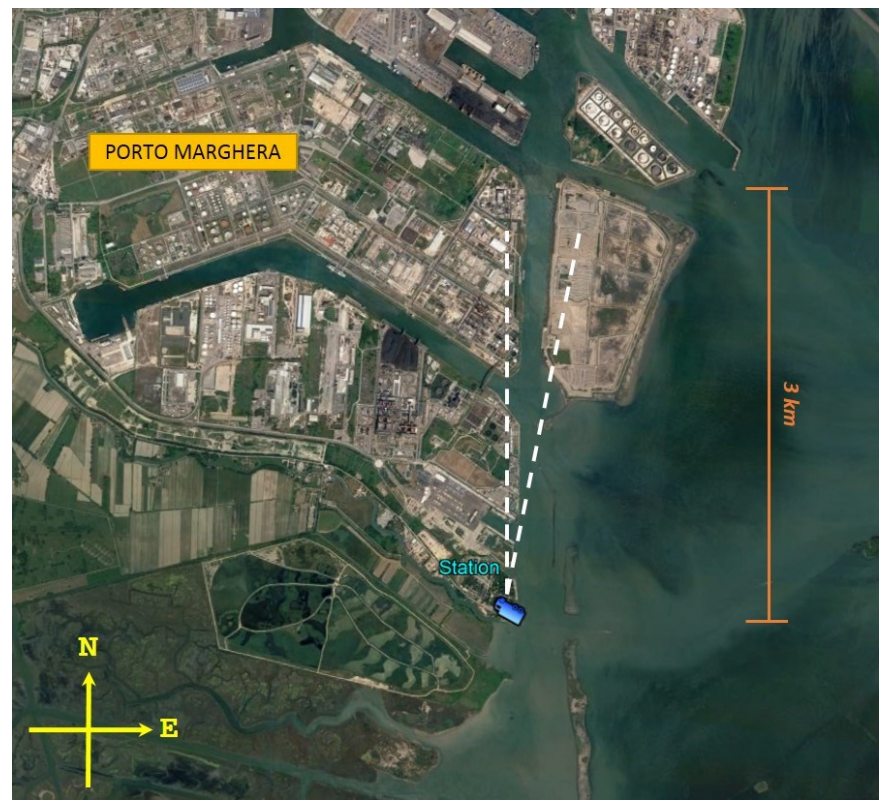

(a)

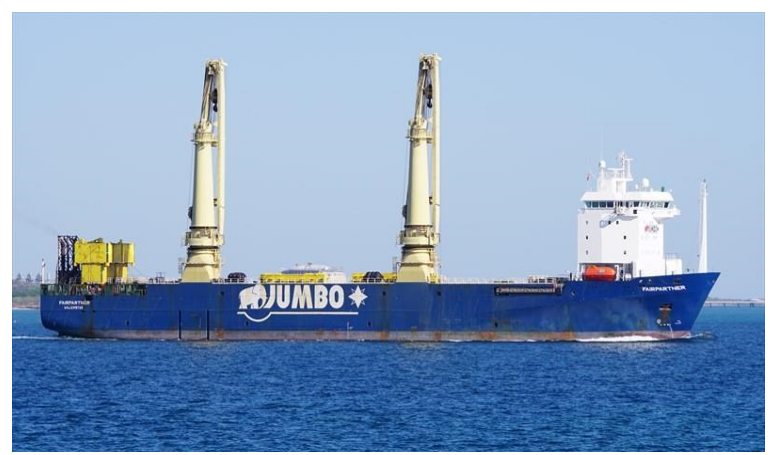

(b)

Fig. 16 Port operations experimental trials. (a) Acquisition geometry. (b) 'Fairpartner' optical photograph [36].

channels are a further confirmation of the diversity gain provided by the GNSS, where the multiple perspectives enhance the possibility to detect a target.

As a proof-of-concept experiment, let us suppose that signals from sats. 2, 3 and 4 only have been acquired. It could be shown that a longer integration time would make possible to reinforce the target power in the individual bistatic links, enabling the target detections in all the long-time bistatic RD maps. However, as highlighted by the analysis in Section IV.A, the number of integrated frames could be in principle traded with multiple baselines to reach comparable detection performance, increasing the robustness of the TMC. We consider here the combination of the three single-frame RD maps after the MSC pertaining the actual target angular values, providing the singleframe multistatic map shown in Fig. 18. As clearly visible, the incoherent summation of the aligned maps lowers the fluctuations of the disturbance background while, at the same time, strengthening the target energy: a clear spot is visible in the figure surrounded by a 'calm' background, so that a high probability of target detection is expected even for low false alarm rate values. In particular, Table IV lists the probability of detection obtained for these short-time RD maps in the bistatic and in the multistatic cases resulting from the theoretical
TABLE III PORT OPERATIONS EXPERIMENTAL CAMPAIGN - TRACKED SATELLITES

\begin{tabular}{llll}
\hline Parameter & & Unit & Value \\
\hline \multirow{4}{*}{ Satellite 1} & Number & - & GSAT0206 \\
& Ranging code & - & E30 $($ E5a-Q) \\
& Azimuth angle & deg & $159.45 \sim 158.95$ \\
& Elevation angle & deg & $49.04 \sim 50.26$ \\
\hline \multirow{5}{*}{ Satellite 2} & Number & - & GSAT0207 \\
& Ranging code & - & E07 (E5a-Q) \\
& Azimuth angle & deg & $-51.83 \sim-51.14$ \\
& Elevation angle & deg & $65.84 \sim 66.84$ \\
\hline \multirow{5}{*}{ Satellite 3 } & Number & - & GSAT0211 \\
& Ranging code & - & E02 $($ E5a-Q) \\
& Azimuth angle & deg & $67.91 \sim 66.36$ \\
& Elevation angle & deg & $49.67 \sim 49.23$ \\
\hline \multirow{5}{*}{ Satellite 4} & Number & - & GSAT0208 \\
& Ranging code & - & E08 $($ E5a-Q) \\
& Azimuth angle & deg & $102.13 \sim 103.46$ \\
& Elevation angle & deg & 56.15 55.26 \\
\hline
\end{tabular}

formulations reported in Section IV.A evaluated for the estimated local SNRs (assuming they correspond to the average values). It can be seen that, even setting a low probability of false alarm, high detection rates can be obtained in the multistatic map, whereas the strong background fluctuations in the bistatic images entail low probability to detect the target. In addition, the $P_{d}^{Q I}$ is close to the maximum $P_{d}^{L R T}$ foreseen by the LRT, thus proving the effectiveness of the QI operator to be used in practical applications. It could be shown that including also sat. 1 , the multistatic $P_{d}$ of detection approaches $100 \%$ for both the QI and LRT detectors (even for $P_{f a}=10^{-5}$ ), thanks to the high SNR level observed in the bistatic map shown in Fig. 17a. Actually, the high SNR observed with sat. 1 allows very high $P_{d}(\approx 99 \%)$ even with the individual bistatic map. This further encourages to consider multistatic geometries with a large number of baselines, because the higher chances to intercept a favorable perspective in terms of bistatic RCS. Even though the target could be detected in this particular bistatic geometry, its localization requires the exploitation of the multiple baselines. The localization results obtained by the proposed approach are shown in the remainder of the section.

Fig. 19a shows the estimated positions of 'Fairpartner' reported in the geographical plane and compared with the AIS ground truth. The short-time $(\mathrm{N}=1)$ maps provided by all the available satellites have been employed, considering successive integration windows with interval $10 \mathrm{~s}$. The good agreement between the actual and estimated track confirms the effectiveness of the localization capability of the technique. In particular, a RMSE of about $38 \mathrm{~m}$ was found, further validating the proposed approach. A further improvement could be achieved considering also an integration over multiple frames: $\mathrm{N}=10$ is chosen here as example. The estimated tracks for the two cases $(\mathrm{N}=1$ and $\mathrm{N}=10)$ are compared in the Cartesian plane (Fig. 19b). An improvement in the track estimation can be observed by exploiting both the space and time domains, as confirmed also by the RMSE reducing at about $24 \mathrm{~m}$.

\section{CONCLUSIONS}

This work explored how the spatial diversity offered by the large number of navigation satellites simultaneously observing each point over the Earth 'surface can be exploited in a GNSS- 


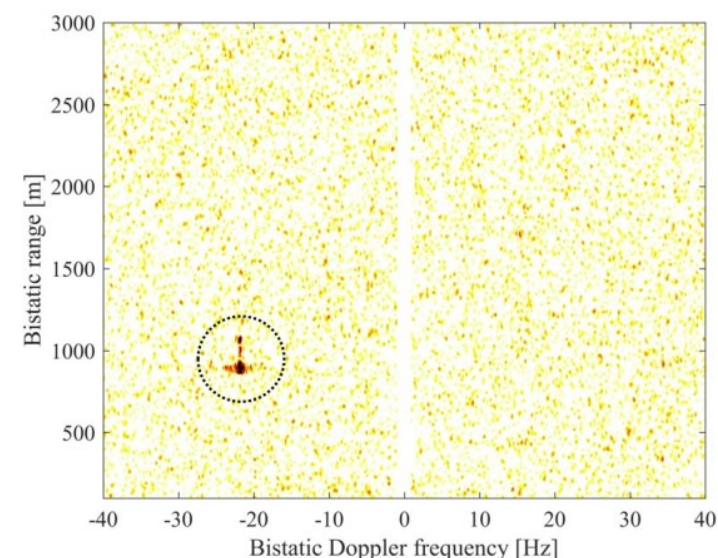

(a)

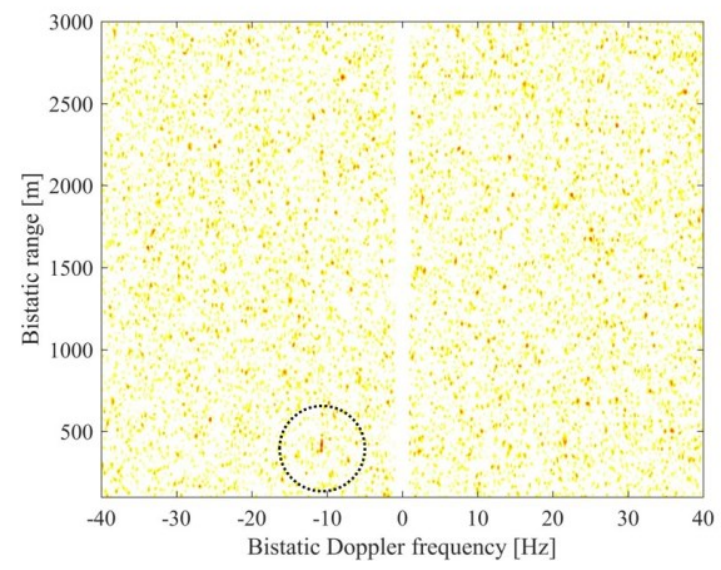

(c)

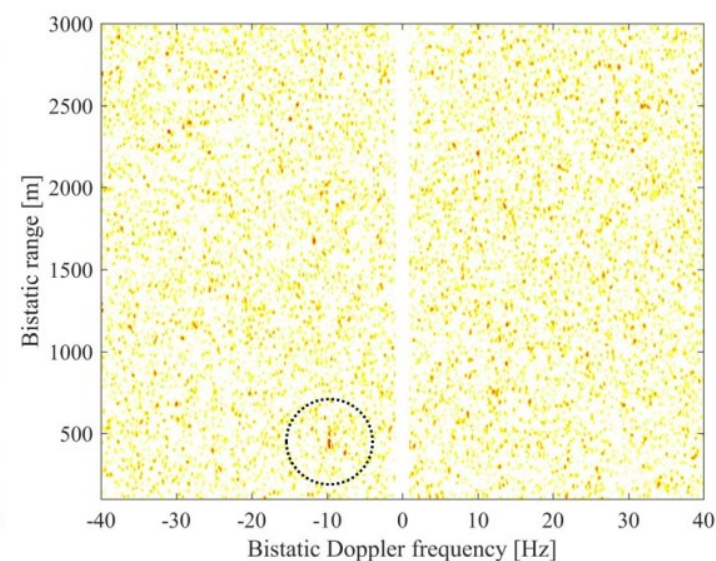

(b)

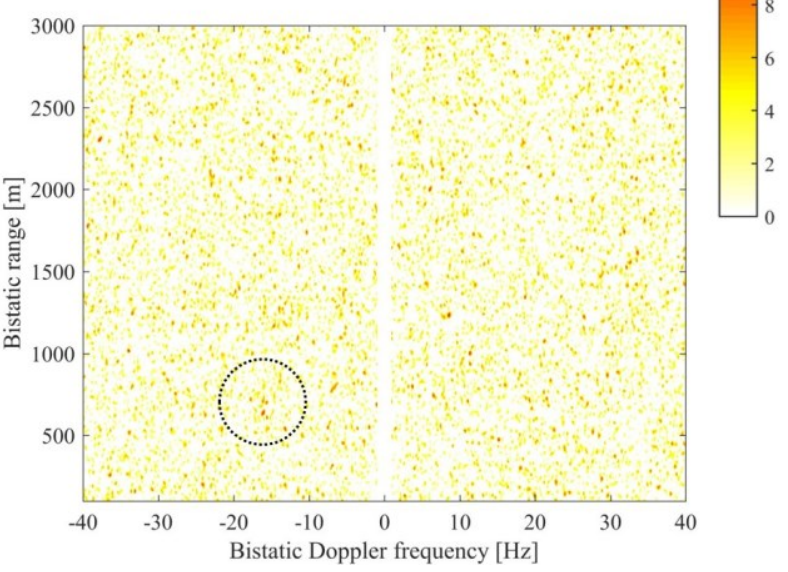

(d)

Fig. 17 'Fairpartner' single frame bistatic RD maps. (a) sat. 1. (b) sat. 2. (c) sat. 3. (d) sat. 4.

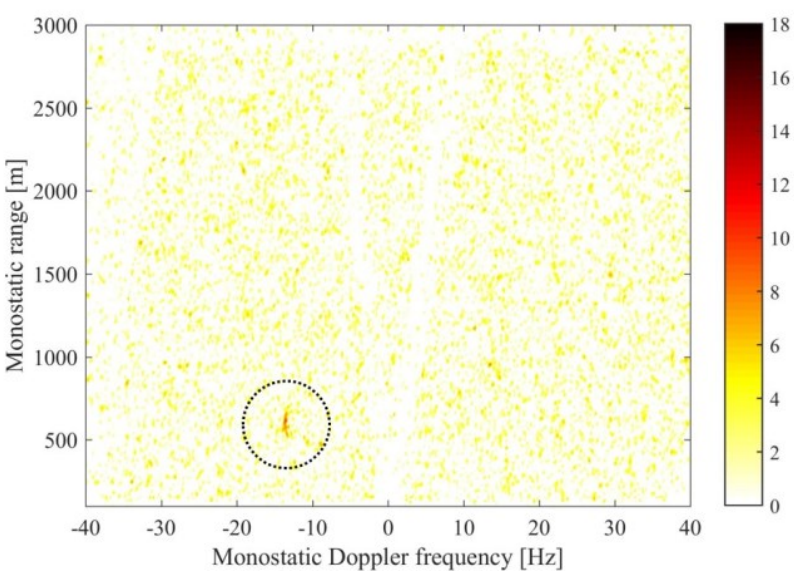

Fig. 18 'Fairpartner' single frame multistatic RD map using satellites $2,3,4$.

based passive radar system for maritime surveillance applications. In particular, a method for enhancing the detection capability of the system is proposed by reinforcing the target power by considering both long integration times and multiple illuminators, thus counteracting the restricted power budget provided by these sources representing the main shortcoming of this technology. By means of a single-stage approach, a joint detection and localization of vessels at sea is demonstrated to be feasible, even for those targets not detectable by means of
TABLE IV ESTIMATED PROBABILITY OF DETECTION FOR 'FAIRPARTNER' SHORT-TIME BISTATIC AND MULTISTATIC RD MAPS

\begin{tabular}{cccccc}
\hline $\boldsymbol{P}_{\boldsymbol{f} \boldsymbol{a}}$ & & sat.2 & sat.3 & sat.4 & $\begin{array}{c}\text { sat.2+sat.3 } \\
\text { +sat.4 }\end{array}$ \\
\hline \multirow{2}{*}{$10^{-3}$} & QI & $66.7 \%$ & $64.3 \%$ & $56.2 \%$ & $95.8 \%$ \\
& LRT & $67.3 \%$ & $64.5 \%$ & $57.2 \%$ & $95.9 \%$ \\
\hline \multirow{2}{*}{$10^{-4}$} & QI & $58.2 \%$ & $55.5 \%$ & $46.4 \%$ & $92.9 \%$ \\
& LRT & $58.4 \%$ & $55.8 \%$ & $46.6 \%$ & $93.0 \%$ \\
\multirow{2}{*}{$10^{-5}$} & QI & $50.9 \%$ & $47.9 \%$ & $38.2 \%$ & $89.6 \%$ \\
& LRT & $51.4 \%$ & $48.5 \%$ & $38.4 \%$ & $89.8 \%$ \\
\hline
\end{tabular}

conventional MTI approaches, by exploiting the inherent multistatic nature of GNSS-based passive radar systems.

A straightforward QI combination rule has been considered for the combination of the RD maps pertaining different satellites. Even though this does not represent the optimum solution because of the different average RCS observed by multiple perspective, it has been demonstrated that it offers a robust approach reaching comparable performance to those provided by the ideal LRT detector.

Moreover, concerning the variation of the target RCS under different bistatic angles, it has to be pointed out that having multiple independent looks at the target is actually a strong benefit in a multistatic radar system. Indeed, even though the returned target power can be very low for a given bistatic 


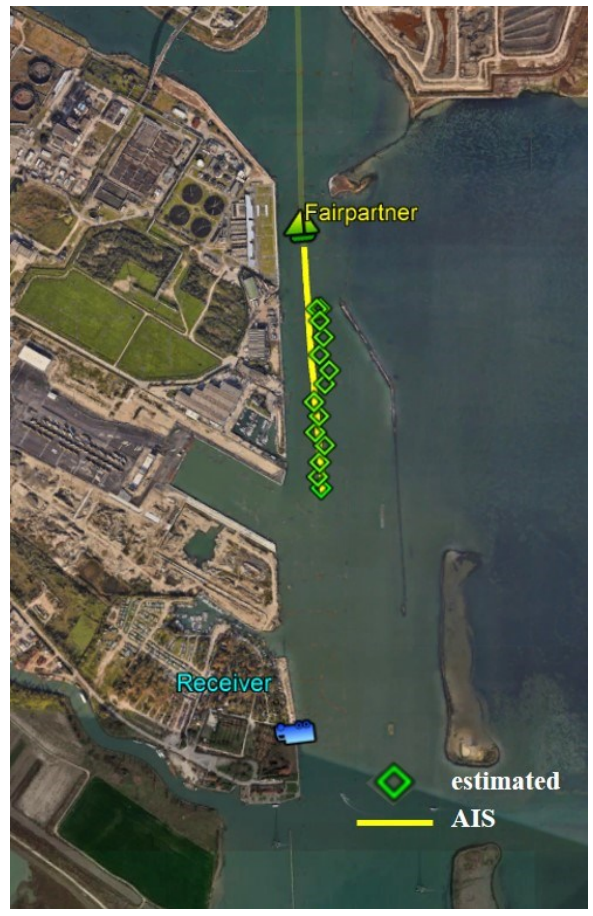

(a)

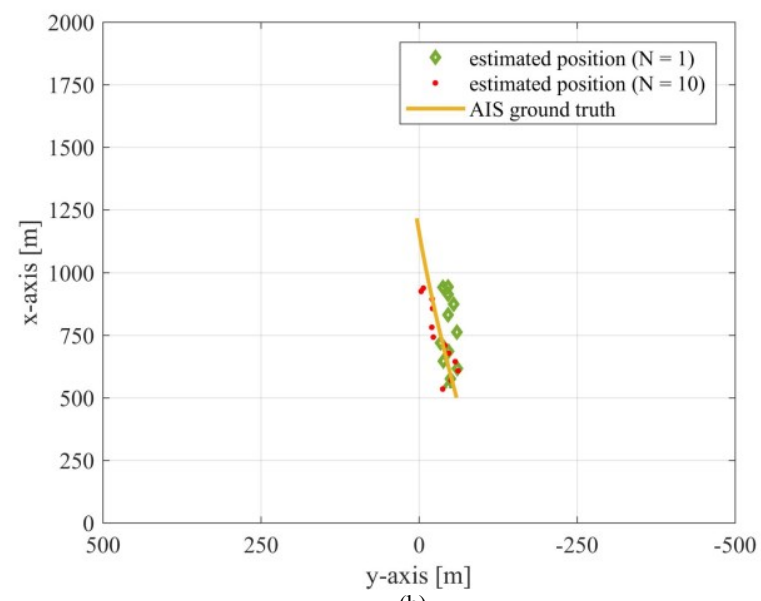

(b)

Fig. 19 'Fairpartner' estimated positions. (a) Geographical plane $(\mathrm{N}=1)$

(b) Cartesian plane.

geometry, considering multiple geometry greatly enhances the chances of observing a strong backscattered energy from another bistatic angle, and this is expected to likely hold for the GNSS multistatic passive radar where widely separated and abundant perspectives are simultaneously available.

As in every multistatic radar system, ghost phenomenon may arise in the case of multiple close targets, affecting the reliability of the system. In this regard, it has to be remarked that the large spatial diversity of the system is expected to provide an inherent ghost rejection capability, which will be subject of future investigations.

Experimental results considering two different scenarios involving vessels of different types supported the theoretical performance analysis, confirmed the effectiveness of the proposed technique to jointly detect and localize vessels at sea despite the strong limitation represented by low EIRP sources employed.
After investigating how to enable the detection and localization capabilities of the system, next stage of research is exploring how the GNSS-based passive radar could retrieve useful information for vessels identification. Possible strategies can rely on the gain in information through observation of a ship under different perspectives, where both the RCS variation and the different spatial resolutions may enable ship features extraction for classification purposes.

\section{APPENDIX A - DERIVATION OF THE LRT DETECTOR}

The binary hypothesis test (24) can be rewritten as

$$
\begin{array}{ll}
\mathcal{H}_{0}: & r_{m, n} \sim \mathcal{C N}\left(0, \sigma_{w}^{2}\right) \\
\mathcal{H}_{1}: & r_{m, n} \sim \mathcal{C N}\left(0, \sigma_{m}^{2}+\sigma_{w}^{2}\right)
\end{array}
$$

According to the Neyman-Pearson criterion, the optimum solution is obtained by comparing the ratio of the likelihood of the received data under $\mathcal{H}_{1}$ over that under $\mathcal{H}_{0}$ to an appropriate threshold $t_{h}$ set to guarantee the desired level of false alarm rate, carrying to the LRT detector, namely

$$
\mathcal{L}=\frac{p\left(\boldsymbol{r} \mid \mathcal{H}_{1}\right)}{p\left(\boldsymbol{r} \mid \mathcal{H}_{0}\right)} \underset{\mathcal{H}_{0}}{\gtrless} t_{h}
$$

where $\boldsymbol{r}=\left[r_{1,-\frac{N}{2}}, \ldots, r_{m,-\frac{N}{2}}, \ldots r_{M,-\frac{N}{2}}, \ldots, r_{1, n}, \ldots, r_{m, n}, \ldots, r_{M, \frac{N}{2}-1}\right]$ is the $1 \times M N$ vector of the received data, $p\left(\boldsymbol{r} \mid \mathcal{H}_{0}\right)$ and $p\left(\boldsymbol{r} \mid \mathcal{H}_{1}\right)$ are their probability density functions under the null and alternative hypothesis, respectively. Assuming both disturbance and target to be independent from frame to frame and from bistatic link to bistatic link, they are given by

$$
\begin{array}{r}
p\left(\boldsymbol{r} \mid \mathcal{H}_{j}\right)=\prod_{m=1}^{M} \prod_{n=-\frac{N}{2}}^{\frac{N}{2}-1} \frac{1}{\sigma_{w}^{2}+j \sigma_{m}^{2}} \exp \left\{-\frac{\left|r_{m, n}\right|^{2}}{\sigma_{w}^{2}+j \sigma_{m}^{2}}\right\} \\
j=0,1
\end{array}
$$

Substituting (A.3) into (A.2) and taking the logarithm yields

$$
\begin{gathered}
-N \sum_{m} \ln \left(\sigma_{w}^{2}+\sigma_{m}^{2}\right)-\sum_{m} \frac{1}{\left(\sigma_{w}^{2}+\sigma_{m}^{2}\right)} \sum_{n}\left|r_{m, n}\right|^{2}+ \\
\mathrm{NM} \cdot \ln \left(\sigma_{w}^{2}\right)+\frac{1}{\sigma_{w}^{2}} \sum_{m} \sum_{n}\left|r_{m, n}\right|^{2}
\end{gathered}
$$

Carrying out the calculus, the test statistic can be written as

$$
\mathcal{L}=\sum_{m} \sum_{n} \frac{\sigma_{m}^{2}}{\sigma_{w}^{2}+\sigma_{m}^{2}}\left|r_{m, n}\right|^{2} \underset{\mathcal{H}_{0}}{\stackrel{\mathcal{H}_{1}}{\gtrless}} t_{h}^{L R T}
$$

where the threshold in (A.5) is a suitable modified version of the threshold in (A.2). Finally, $\frac{\sigma_{m}^{2}}{\sigma_{w}^{2}+\sigma_{m}^{2}}$ can be rewritten as $\frac{S N R_{m}}{S N R_{m}+1}$, so that (A.5) can be equivalently written as (25).

\section{APPENDIX B - PERFORMANCE ANALYIS OF QI AND LRT}

For a generic positive random variable $x$ gamma-distributed with shape parameter $\alpha$ and scale parameter $\beta$, denoted as $x \sim \Gamma(\alpha, \beta)$, the probability density function is given by

$$
p_{X}(x)=\frac{x^{\alpha-1} e^{-\frac{x}{\beta}}}{\Gamma(\alpha) \beta^{N}}, x>0
$$


TABLE B1 PARAMETERS OF THE DISTRIBUTION FUNCTION (B.10) FOR THE EVALUATION OF THE CA AND LRT DETECTORS PERFORMANCE

\begin{tabular}{|c|c|c|c|c|}
\hline$P(\omega)$ & $\beta_{m}$ & $\beta_{\min }$ & $\begin{array}{c}C \\
\text { From (B.7) }\end{array}$ & $\begin{array}{c}\delta_{k} \\
\text { Recursively evaluated by (B.8) and (B.9) }\end{array}$ \\
\hline$P_{q^{\prime} \mid \mathcal{H}_{0}}$ & $\eta_{m} \sigma_{w}^{2}$ & $\eta_{\min } \sigma_{w}^{2}$ & $C_{A}=\prod_{m=1}^{M}\left(\frac{\eta_{\min }}{\eta_{m}}\right)^{N}$ & $\begin{array}{c}\delta_{A_{k+1}}=\frac{1}{k+1} \sum_{i=1}^{k+1} i g_{A_{i}} \delta_{A_{k+1}-i}, k=0,1,2, \ldots \\
g_{A_{k}}=\frac{N}{k} \sum_{m=1}^{M}\left(1-\frac{\eta_{\min }}{\eta_{m}}\right)^{k}, k=1,2, \ldots \\
\delta_{A_{0}}=1\end{array}$ \\
\hline$P_{q^{\prime} \mid \mathcal{H}_{1}}$ & $\eta_{m}\left(\sigma_{m}^{2}+\sigma_{w}^{2}\right)$ & $\eta_{\min }\left(\sigma_{\min }^{2}+\sigma_{w}^{2}\right)$ & $C_{B}=\prod_{m=1}^{M}\left(\frac{S N R_{\min }}{S N R_{m}}\right)^{N}$ & $\begin{array}{c}\delta_{B_{k+1}}=\frac{1}{k+1} \sum_{i=1}^{k+1} i g_{B_{i}} \delta_{B_{k+1-i}}, k=0,1,2, \ldots \\
g_{B_{k}}=\frac{N}{k} \sum_{m=1}^{M}\left(1-\frac{S N R_{\min }}{S N R_{m}}\right)^{k}, k=1,2, \ldots \\
\delta_{B_{0}}=1\end{array}$ \\
\hline$P_{q \mid \mathcal{H}_{1}}$ & $\left(\sigma_{m}^{2}+\sigma_{w}^{2}\right)$ & $\left(\sigma_{\min }^{2}+\sigma_{w}^{2}\right)$ & $C_{C}=\prod_{m=1}^{M}\left(\frac{S N R_{\min }+1}{S N R_{m}+1}\right)^{N}$ & $\begin{array}{c}\delta_{C_{k+1}}=\frac{1}{k+1} \sum_{i=1}^{k+1} i g_{C_{i}} \delta_{C_{k+1}-i}, k=0,1,2, \ldots \\
g_{C_{k}}=\frac{N}{k} \sum_{m=1}^{M}\left(1-\frac{S N R_{\min }+1}{S N R_{m}+1}\right)^{k}, k=1,2, \ldots \\
\delta_{C_{0}}=1\end{array}$ \\
\hline
\end{tabular}

Let us define the random variable $q_{m}=\sum_{n}\left|r_{m, n}\right|^{2}$. From the statistical model (24) [or, equally, (A.1)], it follows that under $\mathcal{H}_{0} q_{m}$ has a probability density function as (B.1) with $\alpha=N$ and $\beta=\sigma_{w}^{2}$, i.e. $q_{m} \sim \Gamma\left(N, \sigma_{w}^{2}\right)$, while under $\mathcal{H}_{1}$, $q_{m} \sim \Gamma\left(N, \sigma_{m}^{2}+\sigma_{w}^{2}\right)$. Further, we define the random variable $q_{m}^{\prime}=\eta_{m} q_{m}$. It follows that $q_{m}^{\prime} \sim \Gamma\left(N, \eta_{m} \sigma_{w}^{2}\right)$ under $\mathcal{H}_{0}$ and $q_{m}^{\prime} \sim \Gamma\left(N, \eta_{m}\left(\sigma_{m}^{2}+\sigma_{w}^{2}\right)\right)$ under $\mathcal{H}_{1}$.

Let us define the random variables $q=\sum_{m} q_{m}$ and $q^{\prime}=$ $\sum_{m} q_{m}^{\prime}$. The $P_{f a}$ for the LRT and the QI are given by

$$
\begin{gathered}
P_{f a}^{L R T}=\int_{t_{h}^{L R T}}^{\infty} p\left(q^{\prime} \mid \mathcal{H}_{0}\right) d q=1-P_{q^{\prime} \mid \mathcal{H}_{0}}\left(t_{h}^{L R T}\right) \\
P_{f a}^{Q I}=\int_{t_{h}^{Q I}}^{\infty} p\left(q \mid \mathcal{H}_{0}\right) d q=1-P_{q \mid \mathcal{H}_{0}}\left(t_{h}^{Q I}\right)
\end{gathered}
$$

where $P(\cdot)$ denotes the distribution function.

For the $P_{d}$, we have

$$
P_{d}^{L R T}=1-P_{q^{\prime} \mid \mathcal{H}_{1}}\left(t_{h}^{L R T}\right)
$$

and

$$
P_{d}^{Q I}=1-P_{q \mid \mathcal{H}_{1}}\left(t_{h}^{Q I}\right)
$$

Therefore, to evaluate the LRT and QI performance we need expressions of $P_{q^{\prime} \mid \mathcal{H}_{0}}, P_{q \mid \mathcal{H}_{0}}, P_{q^{\prime} \mid \mathcal{H}_{1}}$ and $P_{q \mid \mathcal{H}_{1}}$.

Under the null hypothesis, $q$ is the result of the summation of $\mathrm{M}$ identically distributed gamma variates. Therefore $q \sim \Gamma\left(M N, \sigma_{w}^{2}\right)$ and $P_{q \mid \mathcal{H}_{0}}(x)=\frac{\gamma\left(M N, x / \sigma_{w}^{2}\right)}{\Gamma(M N)}$. Replacing in (B.3), (30) is obtained.

Variables $q$ (under $\mathcal{H}_{1}$ ) and $q^{\prime}$ (under both $\mathcal{H}_{0}$ and $\mathcal{H}_{1}$ ) are each one the result of a summation of M gamma-variates with same shape but different scale. The distribution function of the sum of M independent gamma random variables $x_{m} \sim \Gamma\left(\alpha, \beta_{m}\right)$ with $\beta_{m}$ ' not restricted to be identical is given by [37]

$$
P(\omega)=C \cdot \sum_{k=0}^{\infty} \delta_{k} \int_{0}^{\omega} \frac{x^{\rho+k-1} e^{-\frac{x}{\beta_{1}}}}{\Gamma(\rho+k) \beta_{\min }^{\rho+k}} d x, x>0
$$

where $\beta_{\text {min }}=\min _{m} \beta_{m}$ and $\rho=\alpha M ; C$ is equal to

$$
C=\prod_{m=1}^{M}\left(\frac{\beta_{\min }}{\beta_{m}}\right)^{\alpha}
$$

and the coefficients $\delta_{k}$ can be recursively obtained as

$$
\delta_{k+1}=\frac{1}{k+1} \sum_{i=1}^{k+1} i g_{i} \delta_{k+1-i}, k=0,1,2, \ldots
$$

by setting $\delta_{0}=1$ and evaluating the parameters $g_{i}$ by the following rule

$$
g_{k}=\frac{\alpha}{k} \sum_{m=1}^{M}\left(1-\frac{\beta_{\min }}{\beta_{m}}\right)^{k}, \quad k=1,2, \ldots
$$

In (B.6) it can be observed that the integral represents the distribution function of a random variable $x \sim \Gamma\left(\rho+k, \beta_{\text {min }}\right)$ evaluated in $\omega$. Therefore, $P(\omega)$ can be rewritten as

$$
P(\omega)=C \cdot \sum_{k=0}^{\infty} \delta_{k} \frac{\gamma\left(\alpha M^{\omega} / \beta_{\min }\right)}{\Gamma(\rho+k)}, x>0
$$

By using the above equations, $P_{q^{\prime} \mid \mathcal{H}_{0}}, P_{q^{\prime} \mid \mathcal{H}_{1}}$, and $P_{q \mid \mathcal{H}_{1}}$ can be obtained by proper setting of $\alpha$ and $\beta_{m}$. In particular, in all the cases $\alpha=N$ and therefore $\rho=M N$. Table B1 lists the remainder settings to apply to (B.10) to obtain the three distribution functions, where $\sigma_{\min }^{2}=\min _{m} \sigma_{m}^{2}$ and $\eta_{\min }=$ $\frac{S N R_{\min }}{S N R_{\min }+1}$. Replacing the corresponding functions in (B.2) and in (B.4) with $\omega=t_{h}^{L R T}$ and in (B.5) with $\omega=t_{h}^{Q I}$, after some simple manipulations $P_{f a}^{L R T}, P_{d}^{L R T}$, and $P_{d}^{Q I}$ are found as (29), (31) and (32), respectively.

It should be noted that exact evaluation of (B.10) would require the computation of an infinite series. For practical purposes, only the first $K^{\prime}$ coefficient can be computed, where $K^{\prime}$ has to assure an approximation error lower than a desired accuracy [37].

\section{ACKNOWLEDGMENT}

This work has received funding from the European GNSS Agency under the European Union's Horizon 2020 research 
and innovation programme, grant agreement No 641486, "GALILEO-BASED PASSIVE RADAR SYSTEM FOR MARITIME SURVEILLANCE - SpyGLASS", with project consortium composed by Aster S.p.A. (Italy, coordinator), Sapienza University of Rome (Italy), University of Birmingham (UK) and Elettronica GmbH (Germany). The authors would like to thank Aster S.p.A. for providing the synchronization output.

\section{REFERENCES}

[1] M. Martin Neira, S. D'Addio, C. Buck, N. Floury, and R. Prieto-Cerdeira, "The PARIS ocean altimeter in-orbit demonstrator," IEEE Trans. Geosci. Remote Sens., vol. 49, no. 6, pp. 2209-2237, Jun. 2011.

[2] J. F. Marchan-Hernandez et al., "Sea-State Determination Using GNSSR Data," IEEE Geosci. Remote Sens. Lett., vol. 7, no. 4, pp. 621-625, Oct. 2010.

[3] N. Rodriguez-Alvarez et al., "Soil Moisture Retrieval Using GNSS-R Techniques: Experimental Results Over a Bare Soil Field," IEEE Trans. Geosci. Remote Sens., vol. 47, no. 11, pp. 3616-3624, Nov. 2009.

[4] D. Schiavulli, F. Nunziata, G. Pugliano, and M. Migliaccio, "Reconstruction of the Normalized Radar Cross Section Field From GNSS-R Delay-Doppler Map," IEEE J. Sel. Topics Appl. Earth Observ., vol. 7, no. 5, pp. 1573-1583, May 2014.

[5] E. Valencia, A. Camps, J. Marchan-Hernandez, et al., "Ocean surface's scattering coefficient retrieval by Delay-Doppler Map inversion," IEEE Geosci. Remote Sens. Lett., vol. 8, no. 4, pp. 750-754, Jul. 2011.

[6] S. Jin, G. P. Feng, And, S. Gleason, "Remote sensing using GNSS signals: Current status and future directions," Adv. Space. Res., vol. 47, no. 10, pp. 1645-1653, May 2011.

[7] M. Antoniou, and M. Cherniakov, "GNSS-based bistatic SAR: a signal processing view," EURASIP J. Adv. Sign. Process., 2013: 98.

[8] X. He, M. Cherniakov, and T. Zeng, "Signal detectability in SS-BSAR with GNSS non-cooperative transmitters," IEE-Proc. Radar, Sonar Navigat., vol. 152, no. 3, pp. 124-132, Jun. 2005.

[9] M. Antoniou, Z. Hong, Z. Zhangfan, R. Zuo, Q. Zhang, and M. Cherniakov, "Passive bistatic synthetic aperture radar imaging with Galileo transmitters and a moving receiver: Experimental demonstration," IET Proc. Radar, Sonar Nav., vol. 7, no. 9, pp. 985-993, 2013.

[10] Q. Zhang, M. Antoniou, W. Chang, and M. Cherniakov, "Spatial decorrelation in GNSS-based SAR coherent change detection," IEEE Trans. Geosci. Remote Sens., vol. 53, no. 1, pp. 219-228, Jan. 2015.

[11] F. Santi, M. Antoniou, D. Pastina, "Point spread function analysis for GNSS-based multistatic SAR," IEEE Geosci. Remote Sens. Lett., vol. 12, no. 2, pp. 304-308, Feb. 2015.

[12] H. Ma, M. Antoniou, M. Cherniakov, "Passive GNSS-based SAR resolution improvement using joint Galileo E5 signals," IEEE Geosci. Remote Sens. Lett., vol. 12, no. 8, pp. 1640-1644, Aug. 2015.

[13] T. Zeng et al., "Multiangle BSAR Imaging Based on BeiDou-2 Navigation Satellite System: Experiments and Preliminary Results," IEEE Trans. Geosci. Remote Sens, vol. 53, no. 10, pp. 5760-5773, Oct. 2015.

[14] F. Santi, M. Bucciarelli, D. Pastina, M. Antoniou, M. Cherniakov, "Spatial resolution improvement in GNSS-based SAR using multistatic acquisition and feature extraction," IEEE Trans. Geosci. Remote Sens., vol. 54, no. 10, pp. 6217-6231, Oct. 2016.

[15] F. Liu, X. Fan, T. Zhang and Q. Liu, "GNSS-Based SAR Interferometry for 3-D Deformation Retrieval: Algorithms and Feasibility Study," IEEE Trans. Geosci. Remote Sens, vol. 56, no. 10, pp. 5736-5748, Oct. 2018.

[16] V. Kock and R. Westphal, "New approach to a multistatic passive radar for air/space defense," IEEE Aerosp. Electron. Syst. Mag., vol. 10, no. 11, pp. 24-32, Nov. 1995.

[17] E. P. Glennon, A. G. Dempster, C. Rizos, "Feasibility of air target detection using GPS as a bistatic radar," J. Global Positioning Syst., vol. 5, no. 1-2, pp. 119-126, 2006.

[18] I. Suberviola, I. Mayordomo, and, J. Mendizabal, "Experimental results of air target detection with a GPS forward-scattering radar," IEEE Geosci. Remote Sens. Lett., vol. 9, no. 1, pp. 47-51, Jan. 2012.
[19] H. D. Griffiths and C. J. Baker, "Passive coherent location radar systems. Part I: Performance prediction," IEE-Proc. Radar, Sonar Navigat., vol. 152, no. 3, pp. 153-159, Jun. 2005.

[20] M. Clarizia, P. Braca, C. S. Ruf and P. Willett, "Target detection using GPS signals of opportunity," 2015 18th International Conference on Information Fusion (Fusion), Washington, DC, 2015, pp. 1429-1436.

[21] A. Di Simone, H. Park, D. Riccio, and A. Camps, "Sea target detection using spaceborne GNSS-R delay-Doppler maps: Theory and experimental proof of concept using TDS-1 data," IEEE J. Sel. Topics Appl. Earth Observ., vol. 10, no. 9, pp. 4237-4255, Sep. 2017.

[22] A. Di Simone, P. Braca, L. M. Millefiori and P. Willett, "Ship detection using GNSS-reflectometry in backscattering configuration," 2018 IEEE Radar Conference (RadarConf18), Oklahoma City, OK, 2018, pp. 15891593.

[23] H. Ma, M. Antoniou, D. Pastina, F. Santi, F. Pieralice, M. Bucciarelli, M. Cherniakov, "Maritime moving target indication using passive GNSSbased passive radar," IEEE Trans. Aerosp. Electron. Syst., vol. 54, no. 1, pp. 115-130, Feb. 2018.

[24] D. Pastina, F. Santi, F. Pieralice, M. Bucciarelli, H. Ma, D. Tzagkas, M. Antoniou, M. Cherniakov, "Maritime moving target long time integration for GNSS-based passive bistatic radar", IEEE Trans. Aerosp. Electron. Syst., vol. 54, no. 6, pp. 3060-3083, Dec. 2018.

[25] Z. Li, F. Santi, D. Pastina, P. Lombardo, "Passive radar array with lowpower satellite illuminators based on fractional Fourier transform," IEEE Sensors Journal, vol. 17, no. 24, pp. 8378-8394, Dec. 2017.

[26] E. Fisher, et al., "Spatial diversity in radars - Models and detection performance," IEEE Trans. Sign. Process., vol. 54, no. 3, pp. 823-838, Mar. 2006.

[27] H. Ma, M. Antoniou, A.G. Stove, J. Winkel, and M. Cherniakov, "Maritime moving target localization using passive GNSS-based multistatic radar," IEEE Trans. Geosci. Remote Sens, vol. 56, no. 8, pp. 4808-4819, Aug. 2018.

[28] G. Mellen, M. Patcher, J. Racquet, "Closed-form solution for determining emitter location using time difference of arrival measurements," IEEE Trans. Aerosp. Electron. Syst., vol. 39, no. 3, pp. 1056-1058, Jul. 2003.

[29] D.E. Hack, L. K. Patton, A. D. Kerrick, and M. Saville, "Direct Cartesian detection, localization, and de-ghosting for passive multistatic radar," Proc. IEEE $7^{\text {th }}$ Sensor Array and Multichannel Processing Workshop, pp. 45-48, 2012.

[30] F. Pieralice, D. Pastina, F. Santi, M. Bucciarelli, "Multi-transmitter ship target detection technique with GNSS-based passive radar," Proc. Int. Conf. on Radar Syst., Belfast, Oct. 2017, pp. 1-6.

[31] F. Santi, F. Pieralice, D. Pastina, "Multistatic GNSS-based passive radar for maritime surveillance with long integration times: experimental results," Proc. IEEE RadarConf18, Oklahoma City, OK, Apr. 2018, pp. 1260-1265.

[32] D. Pastina, M. Sedhei, D. Cristallini, "Geostationary satellite based passive bistatic ISAR for coastal surveillance," Proc. IEEE Radar Conference, Washignton, DC, USA, May 2010.

[33] D. Zhu, and Z. Zhu, "Range Resampling in the Polar Format Algorithm for Spotlight SAR Image Formation Using the Chirp z-Transform," IEEE Trans. Sign. Process., vol. 55, no. 3, pp. 1011-1023, Mar. 2007.

[34] D. Baumgarten, "Optimum detection and receiver performance for multistatic radar configurations," Proc. IEEE Int. Conf. Acoustics, Speech, and Signal Proc., 1982, pp. 359-362.

[35] T. Kelso, CelesTrack, Public domain satellite tracking website, 2010 [Online]. Available: http://celestrack.com/

[36] Marine Traffic. [Online]. Available: www.marinetraffic.com

[37] P. G. Moschopoulos, "The distribution of the sum of independent Gamma random variables," Ann. Inst. Statist. Math., vol. 37, no. 1, Dec. 1985, pp. 541-544.

[38] SPyGLASS - GALILEO-BASED PASSIVE RADAR SYSTEM FOR MARITIME SURVEILLANCE, 2015. [Online]. Available: www.spyglassproject.eu. 